\title{
A Numerical Study of the Feedback Mechanisms of Hurricane-Environment Interaction on Hurricane Movement from the Potential Vorticity Perspective
}

\author{
Chun-Chieh Wu \\ Department of Atmospheric Sciences, National Taiwan University, Taipei, Taiwan \\ YOSHIO KURIHARA \\ Geophysical Fluid Dynamics Laboratory, NOAA, Princeton University, Princeton, New Jersey
}

(Manuscript received 6 October 1995, in final form 15 February 1996)

\begin{abstract}
The interaction between a hurricane and its environment is studied by analyzing the generation and influence of potential vorticity (PV) from the Geophysical Fluid Dynamics Laboratory hurricane model analysis system. Two sets of numerical experiments are performed: one with and the other without a bogused hurricane vortex in the initial time, for cases of Hurricanes Bob (1991), Gilbert (1988), and Andrew (1992).

The PV budget analysis of Bob shows that the condensational heating within the vortex redistributes the PV, causing a PV sink in the upper part of the vortex and a PV source in the lower part. This tendency is compensated for largely, but not entirely, by the upward transport of high-PV air from the lower levels to the upper levels. The net effect contributes to the increase of the negative upper-level PV anomaly during the vortex intensification period. This result indicates that the diabatic heating effect plays a crucial role in the evolution of the PV field in hurricanes. It also suggests the importance of accurate representation of the heating profile in hurricane models.

It is shown that the negative upper-level PV anomaly is spread out by the upper-level outflow and the largescale background flow. The impact of the spread of the negative upper PV anomaly to the storm is quantitatively evaluated by computing the nonlinear balanced flow associated with the PV perturbation. Notable contribution to the steering of the storm from the upper-level PV anomaly is found. The result supports the theory advanced by Wu and Emanuel conceming the effect of the upper negative PV anomaly on hurricane motion. This study aiso indicates the need of enhanced observation and accurate analysis and prediction in the upper troposphere in order to improve hurricane track forecasting.
\end{abstract}

\section{Introduction}

A hurricane is a localized, yet robust, vortex. Its strong circulation can substantially change the surrounding environmental flow field, which can in turn affect the evolution of the track, intensity, and structure of the hurricane. This interaction between the tropical cyclone and its environment is nonlinear. A better knowledge of how the hurricane and its environment interact is an important part of improving both our understanding of hurricane dynamics and our ability to forecast actual storms.

Previous studies have investigated how the largescale flow fields affect the track, intensity (e.g., Molinari et al. 1995), and structure (M. Bender 1995, personal communication) of the hurricane, or how the storm circulation changes the environmental flow field (e.g., Ross and Kurihara 1995). These studies consider

Corresponding author address: Dr. Chun-Chieh Wu, Department of Atmospheric Sciences, National Taiwan University, 61, Ln. 144, Sec. 4, Keelung Rd., Taipei 10772, Taiwan.

E-mail: cwu@typhoon.as.ntu.edu.tw only a one-way interaction between the storm and its environment. In this work, the two-way hurricane-environment interaction is studied, that is, how the change of the environment due to the storm feeds back to affect the storm's track. Results are compared to current theories on the dynamics of hurricane motion, including the $\beta$ effect (e.g., Chan and Williams 1987; Fiorino and Elsberry 1989) and the effect of upper-tropospheric negative potential vorticity (PV) anomaly (Wu and Emanuel 1993, hereafter WEM93). To investigate the above theories, potential vorticity diagnostics of the Geophysical Fluid Dynamics Laboratory (GFDL) hurricane model analysis system are employed.

Some background for this study is reviewed in section 2. Section 3 describes the model and methodology. The results are presented in section 4 . The summary appears in section 5 .

\section{Background review}

\section{a. Theories on hurricane motion}

A hurricane is analogous to a vortex in a mean stream flow. In the case of a hurricane, a cyclonic vor- 
tex moves toward a region where amplification of cyclonic vorticity is favored. This process is dominated by vorticity advection by the vertically averaged advecting flow at the vortex center, the "steering current." Due to the paucity of observations and the existence of strong azimuthal winds in and around hurricanes, deriving the vertically averaged instantaneous flow through the storm center is difficult. It is conventional to approximate the hurricane steering flow from the annular and pressure-weighted vertical means of the tropospheric horizontal large-scale flow surrounding the storm (e.g., Chan and Gray 1982). However, this estimate may have excluded the component of the hurricane advecting flow associated with some smallerscale or asymmetric dynamical features.

The steering flow is often associated with such systems as the subtropical high, the monsoon trough, and other synoptic features (e.g., the upper-level trough or ridge). Our understanding of hurricane movement primarily relies on our ability to predict the evolution of those large-scale systems surrounding the hurricane vortex. However, the hurricane's strong circulation can also affect its background fields, and this change may later influence the storm's advecting current. It is important to know whether the change of the environment by the storm can feed back to influence the hurricane's own evolution, particularly to its steering.

A well-known theory of a feedback mechanism for hurricane movement concerns the so-called $\beta$ effect. Due to the variation of the Coriolis parameter across the tropical cyclone, the cyclonic circulation associated with the hurricane tends to advect background planetary vorticity gradient and results in a pair of dipole vorticity gyres that eventually advect the storm poleward and westward, even though initially no ambient steering current exists. This theory has been confirmed by many numerical models, most of which investigated the evolution of a barotropic vortex on a beta plane in an quiescent environment (e.g., Chan and Williams 1987; Fiorino and Elsberry 1989). These models are good examples of applying the fundamental conservation principles in geophysical fluid dynamics to advance our understanding on the movement of the hurricane-like vortex on the rotating atmosphere.

Recently, M. Bender (1995, personal communication) took the time average of the asymmetric flow field from an idealized hurricane integration of the GFDL hurricane model and was also able to find distinct $\beta$ gyres. However, as nature is much more complicated than what the idealized model or idealized hurricane can describe, detecting the $\beta$ effect from observations is far more difficult (Ulrich and Smith 1991). Field experiments [e.g., TCM90; see Elsberry et al. (1990)] have been conducted to collect better data for investigating the $\beta$ effect. Unfortunately, the observational results are still inconclusive. It is still debatable (Reeder et al. 1991, 1992; Holland 1992) whether it will be possible to use data such as from TCM90 to accomplish the goal of detecting the $\beta$ effect. Nevertheless, the $\beta$ effect has already been incorporated into the initialization procedure for some dynamical hurricane models [e.g., the GFDL hurricane model; Bender et al. (1993)].

On the other hand, baroclinic models have been developed for examining a more generalized notion of the $\beta$ effect (e.g., Shapiro 1992; WEM93). Shapiro used a three-layer model to evaluate the asymmetric evolution of a hurricane and its interaction with the largescale environment that contains a mean vertical shear. He showed that vortices may move in response to asymmetric vorticity advection due to the presence of the background PV gradient in a manner similar to the vortex motion resulting from the planetary vorticity gradient.

In WEM93, two points regarding the applicability of $\beta$ effect were emphasized. First, real tropical cyclones are strongly baroclinic, consisting of strong cyclonic vorticity surmounted by a broad area of upper-level anticyclonic flow. Second, the actual distribution of the PV gradient in the troposphere is quite nonuniform. Indeed, the magnitude of the PV gradient is concentrated near the tropopause. WEM93 suggested that hurricane motion can be impacted substantially by these two observed features.

\section{b. PV perspective}

Potential vorticity methods have proven useful in understanding synoptic- and large-scale midlatitude dynamics (Hoskins et al. 1985) and are becoming more widely applied to tropical systems. WEM93 and Wu and Emanuel (1994, hereafter WEM94) have shown that PV concepts can be usefully applied to the understanding of hurricane movement and, to some extent, to hurricane structure, even though there exist strong sources of PV. WEM93 indicated that hurricanes have strong sources of heating near their centers. From a PV point of view, this source tends to redistribute the PV in the hurricane by creating a sink of PV in the upper troposphere above the storm. As the hurricane evolves in time, the negative PV anomaly above the hurricane expands due to the outflow from the hurricane. Since this negative PV anomaly can have an extensive horizontal scale, presumably its associated flow can penetrate downward to the lower troposphere and interact with the hurricane vortex. If there is a background vertical shear, the baroclinic hurricane vortex would be tilted, and the negative upper PV anomaly can produce a flow projecting down to the lower troposphere, which can advect the hurricane vortex to the left of the shear vector.

Based on observations that PV is well mixed in the troposphere (Davis and Emanuel 1991), WEM93 and WEM94 constructed a simple quasigeostrophic contour dynamics model that has a background shear but no background PV gradient, and they showed that the interaction of the baroclinic hurricane vortex with the 
shear leads to storm motion and a strong deformation of the upper-level potential vorticity, resulting in a jetlike outflow structure. Indeed, WEM93 indicated that the flow field associated with the upper-tropospheric negative PV anomaly generated by the hurricane can steer the storm to the left of the background shear vector.

In addition, Wu and Emanuel (1995a,b, hereafter WEM95a,b) applied the PV diagnostics to evaluate the hurricane's advecting (steering) flow from the final global analyses of the National Meteorological Center (NMC), archived on a $2.5^{\circ} \times 2.5^{\circ}$ latitudelongitude grid. They showed that the nonlinear balanced flow is a good approximation to the NMC's analyzed flow field, suggesting that their PV diagnostics are valid. By examining the balanced flow at the hurricane center, WEM95a,b were able to identify which PV perturbation has the most influence on hurricane movement. They also defined the hurricane advecting flow as the balanced flow (in the center of the storm) associated with the whole PV in the troposphere, except for the PV anomaly of the hurricane itself. Their results showed that the resulting steering wind is a very good approximation to the real storm motion.

WEM95a,b also compared their results to previous theories on hurricane motion. They found that some negative PV anomaly was generated above the storm, and its location coincided well with the storm position. The PV anomaly was also observed to be advected to the downshear side relative to the storm center. When the piecewise PV inversion was performed, the balanced flow associated with the negative upper-level PV anomalies was shown to have a substantial effect in advecting Hurricane Bob (1991). However, there is no definite way of distinguishing whether the negative anomalies were generated by the presence of the storm or were due to other dynamical advection process in the upper troposphere.

On the other hand, no conclusive evidence of a $\beta$ gyre structure was found in WEM95a,b. Apparently detection of this structure was handicapped by the deficiency of the NMC data, namely, the relatively coarse resolution $\left(2.5^{\circ} \times 2.5^{\circ}\right)$ and the failure to capture the storm's actual intensity. WEM95a,b suggested that better data are needed to further investigate this problem.

Since the GFDL hurricane model has been shown (Kurihara et al. 1990, 1993, 1995; Bender et al. 1993) to perform well in forecasting the hurricane track and, to a certain degree, the hurricane structure and intensity, the high-resolution GFDL model forecast can be valuable for investigating the aforementioned theories. In addition, the three-dimensional numerical model allows a controlled means of identifying the hurricane's impact on the environmental flow change. For these reasons, PV diagnostics of the GFDL hurricane model analysis system are employed to further examine the above theories.

\section{Methodology}

\section{a. Model description}

The GFDL multiply nested movable mesh model originally described by Kurihara and Bender (1980), with additional model details presented in Tuleya et al. (1984), Bender et al. (1987), and Bender et al. (1993, hereafter BRTK), with the new initialization scheme (Kurihara et al. 1995, hereafter KBTR) and with the implementation of the radiation parameterization ( $\mathrm{Tu}-$ leya 1994), is used for the model hurricane forecasts. This primitive equation model is formulated in latitude, longitude, and sigma coordinates, with 18 levels in the vertical (Table 1 of Kurihara et al. 1990). The integration domain is $75^{\circ}$ latitude by $75^{\circ}$ longitude, with a triply nested grid system of resolution of $1^{\circ}, 1 / 3^{\circ}$, and $1 / 6^{\circ}$ (Table 1 of BRTK). The outermost domain ranges from $10^{\circ} \mathrm{S}$ to $65^{\circ} \mathrm{N}$ in the meridional direction and varies in the zonal direction, depending on the storm's location at each forecast time.

The model physics include a cumulus parameterization scheme described by Kurihara (1973), with some modifications (Kurihara and Bender 1980, appendix C): a Monin-Obukhov framework for the interactions at the surface and the Mellor and Yamada (1974) level2 turbulence closure scheme for the vertical diffusion, with a background diffusion coefficient added. In addition, as described by Tuleya (1994), the Schwarzkopf and Fels (1991) infrared and Lacis and Hansen (1974) solar radiation parameterizations are incorporated, including interactive radiative effects of clouds and a diurnal radiation cycle, along with land surface temperature computed by an energy equation containing a soil layer. In this version of the GFDL hurricane model the sea surface temperature is specified and held fixed to the initial distribution throughout the integration.

\section{b. Experimental design}

We adopt the same experimental strategy as used by Ross and Kurihara (1995, hereafter RK). The storm's influence on its environment is identified by comparing two GFDL hurricane model forecasts from initial conditions that differ only in the presence or absence of a hurricane vortex. To evaluate this hurricane-environment interaction, two sets of 72 -h numerical experiments with different initial conditions are conducted using the GFDL hurricane model.

The first experiment (hereafter, referred to as the "hurricane" experiment) starts with an initial condition that is determined from the hurricane model initialization scheme described in KBTR using the NMC T126 global analyses as the initial input data. Using a simple smoothing technique (i.e., 1-2-1 filter, as described in BRTK), the background mean flow is first calculated in KBTR's system. The region containing the perturbation flow field associated with the coarse- 


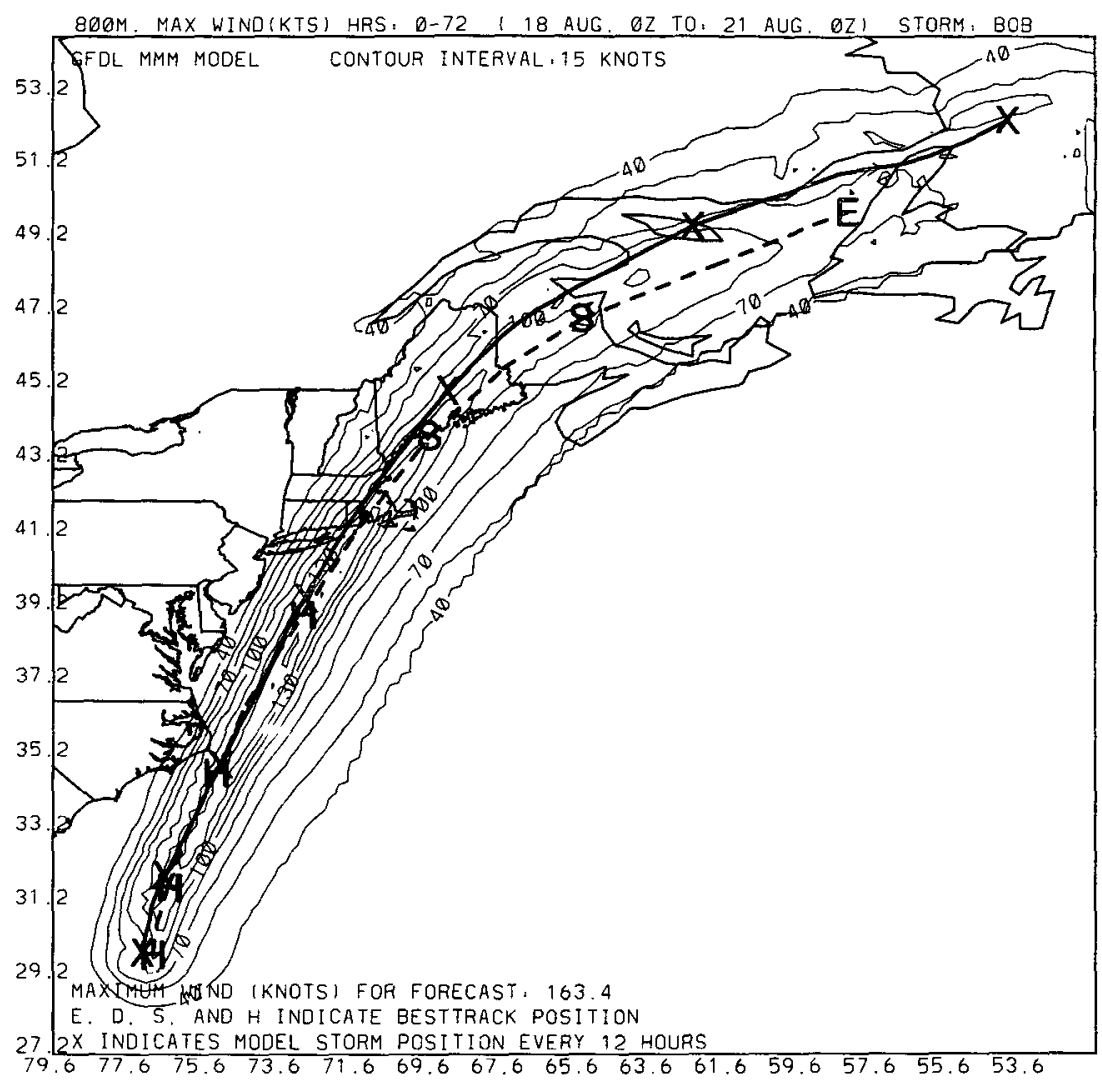

FIG. 1. Hurricane Bob's forecasted track from the GFDL hurricane model (solid) and best track analysis (dashed) from 0000 UTC 18 August to 0000 UTC 21 August 1991. The model storm positions are indicated as " $\mathrm{X}$ " and the best tracks as " $E$ " (extratropical storm), " $D$ " (tropical depression), "S" (tropical storm), or " $\mathrm{H}$ " (hurricane) every $12 \mathrm{~h}$. The model maximum surface wind is shown with a contour interval of $10 \mathrm{kt}$.

resolution hurricane vortex resolved by the National Centers for Environmental Prediction (NCEP, formerly the National Meterological Center) global analyses is then identified, and the hurricane's perturbation field is removed. Using the optimal interpolation and merging scheme, the background environmental field is reconstructed across the local region of the hurricane, while the environmental flow field outside of the storm domain is unchanged. According to KBTR, this method successfully removes the hurricane's cyclonic main circulation while retaining some important environmental flow structure.

In the next step of the initialization system of KBTR, both the symmetric and asymmetric components of the specified vortex are generated. First, an axisymmetric version of the GFDL hurricane model is integrated to force the tangential wind toward a target flow field that resembles the observed hurricane wind profile obtained from the hurricane message and the reconnaissance report. Second, using the obtained tangential wind distribution as input, a simplified version of a barotropic model (Ross and Kurihara 1992) is run in order to produce an asymmetric flow that mimics the $\beta$ effect. The combination of the above axisymmetric and asymmetric flow fields generates a model-consistent and better-resolved hurricane vortex, which is reinserted into the environmental flow field at the correct position to produce the initial condition. Using this flow field, a static initialization is then employed to recover the mass field. KBTR has demonstrated improvement of the performance of GFDL hurricane model forecasts after employing this hurricane initialization scheme.

The initial condition for the second experiment (hereafter, referred to as the "nonhurricane" experiment) is identical to the first one, except that after the analyzed vortex is removed no "bogused" storm is inserted. Therefore, the initial condition of the nonhurricane experiment represents a flow field that has the same environment as the hurricane experiment except for missing the local region of the hurricane circulation. For convenience in comparing the hurricane and nonhurricane experiment, as discussed by RK, the inner meshes of the nested mesh model in the nonhurricane integration are prescribed to move according to the 
mesh movement in the corresponding hurricane integration.

In the present experimental design, the difference in fields outside the storm region between the hurricane and nonhurricane experiments represents the difference in the environmental fields, while that within the storm region results from both the difference in the environmental fields and the presence of a vortex in the hurricane experiment. The environmental field difference, which does not exist anywhere at the initial time, is caused by the hurricane vortex and can spread. Thus it is assumed that any differences in the dynamic fields that develop beyond the local storm region during these two model integrations represent the accumulated impact of the storm on the environment. To make this assumption as safe as possible, we need to choose cases where the model storm forecasts are sufficiently accurate in predicting the actual storm and atmospheric evolution so that the model-predicted influence by the hurricane is also representative of the actual interactions. Even though we do not know the validity of the nonhurricane integration, our filtering of the storm vortex should be justified as long as the nonhurricane integrations yields physically reasonable large-scale fields while not developing a hurricane.

The integrations presented in this study are run for $72 \mathrm{~h}$ using the lateral boundary values specified from the forecasts of the NMC global spectral model, linearly interpolated in time to hourly values. The model solution is then forced toward the next future hourly values at each time step using the lateral boundary forcing scheme of Kurihara et al. (1989). Even though the model simulation is performed after the storm event, it can be regarded as a "forecast" because the integration takes the same initial and boundary data as those used for real-time storm forecast in the GFDL hurricane prediction system.

\section{c. PV analysis}

After integration of the two experiments using the GFDL hurricane model, following the methodology described in WEM95a, we employ potential vorticity diagnostics on the model output. For the analyses employed, all data have been interpolated from the nested grids to a uniform grid of 1-degree resolution. This resolution may not be sufficient to capture the detailed hurricane structure (such as its eye and the maximum PV value) but it should be appropriate for our study of the hurricane--environment interaction. In our PV diagnostics, we regard the output from the nonhurricane simulation as the background mean field and the difference between the hurricane and nonhurricane experiments as the perturbation (or anomaly) field, which should approximately represent the storm's direct influence on its environment. This approximation should be valid as long as the effect from the nonlinear feedback is of secondary importance during the integration.
In this work, we shall focus on following the evolution of the PV perturbation (anomaly) to understand the hurricane's impact on the environment. Through the use of the piecewise PV inversion under the nonlinear balanced condition (same method as used in WEM95a,b), we are able to obtain the flow field associated with any PV anomalies and to quantitatively examine the interactions between those anomalies. We also investigate the influence of each anomaly that is caused by the storm itself on the storm movement. The results will be compared to aforementioned theories on hurricane motion.

\section{Results}

Three cases [Hurricanes Gilbert (1988), Bob (1991), and Andrew (1992)] have been studied. The main reasons for choosing these cases are because these storms were close to the United States, where a more detailed observational network is available and the data quality may be better surrounding the storm, and because the GFDL hurricane model had produced good track and intensity forecasts for these cases. In this paper, the results for Bob are focused and are presented in detail, while the results from the other two cases are briefly discussed.

\section{a. Overview of model performance}

Hurricane Bob originated from the remnants of a frontal trough just south-southeast of Bermuda on 12 August 1991. The disturbance was named a tropical depression at 0000 UTC on 16 August near the Bahamas and was then upgraded to Tropical Storm Bob later on the same day, when it was located about $250 \mathrm{~km}$ (135 $\mathrm{n} \mathrm{mi}$ ) northeast of Nassau. The storm reached hurricane strength on 17 August, about $415 \mathrm{~km}$ (225 $\mathrm{n}$ mi) east of Daytona Beach, Florida. Bob then accelerated, turning toward the north and then north-northeast. Bob continued intensifying and reached its maximum intensity, characterized by $51 \mathrm{~m} \mathrm{~s}^{-1}$ sustained winds and a 950-mb central pressure on 19 August, when it was located $185 \mathrm{~km}(100 \mathrm{n} \mathrm{mi})$ east-southeast of Norfolk, Virginia. The storm weakened while moving to the north-northeast over cooler waters off the mid-Atlantic coast. It made landfall at Newport, Rhode Island, about 1800 UTC 19 August. Bob next moved across Rhode Island and Massachusetts while continuing to weaken. The storm made final landfall as a tropical storm near Rockland, Maine, at 0130 UTC 20 August and eventually evolved into an extratropical cyclone over the Gulf of St. Lawrence.

The 72-h GFDL hurricane model integration of Bob was initialized at 0000 UTC 18 August 1991, when Bob was located about $450 \mathrm{~km}$ southeast of Charleston, South Carolina. Figure 1 shows the analyzed best track and the model's predicted storm track and maximum surface wind distribution. In the first $36 \mathrm{~h}$ of the fore- 
cast, the forecast track is almost identical to the best track. The prediction of the landfall position is very close to the actual landfall location near Newport, Rhode Island. After landfall, the model storm moves somewhat faster and to the north of the best track. Over all, the model's forecast of Bob's track is remarkably good.

Although the skill from hurricane intensity forecast using the current GFDL hurricane model is limited, in this case there is some indication of skill in the intensity forecast, as can be seen from the comparison of the maximum surface wind between the model and observations (Fig. 2). The NMC global (T-126) analyses resolved Bob's intensity only up to a maximum surface wind of $20 \mathrm{~m} \mathrm{~s}^{-1}$. But after the bogusing, the observed maximum wind of $33 \mathrm{~m} \mathrm{~s}^{-1}$ has been restored at the initial time of the model integration. The model overforecasts Bob's intensity by about $20 \%$ in the first 24 $\mathrm{h}$, but it is able to predict the tendency of the intensity change later on.

The time evolution of the three-dimensional wind field (not shown) also clearly indicates a classic hurricane structure in Bob. It includes a strong horizontal azimuthal flow rotating around Bob throughout the troposphere below $200 \mathrm{mb}$. It also displays a typical transverse hurricane secondary circulation, containing the lower-level inflow spiraling toward the storm center, strong updrafts near the center, and divergent outflow in the upper troposphere.

We also compare the synoptic features from the model output to the NMC global analyses during the forecast period (i.e., from 0000 UTC August 18 to 0000 UTC August 21 1991). The model forecast accurately predicts the synoptic-scale environment near the storm (not shown), including the approach of a trough from the central United States, and the evolution of the high pressure system over the western Atlantic. The combination of these two systems contributes to a distinct south-southwesterly flow near Bob's center that advects Bob north-northeastward through the whole integration period. Over all, this can be regarded as a good simulation, and the quality of this model output is good enough to provide us more insight into the hurricane-environment interaction. In particular, the nonhurricane integrations also produce physically reasonable large-scale fields while not developing a hurricane. As mentioned in section $3 b$, even though we do not know the exact validity of the nonhurricane integration, the above findings suggest that the removal of Bob's main vortex in the nonhurricane simulation should be justified, and the PV anomalies deduced from the difference of PV between the hurricane and nonhurricane simulations should be justified as well.

\section{b. PV evolution}

Ross and Kurihara (1994) examined the change of temperature, wind, and sea level pressure fields due to

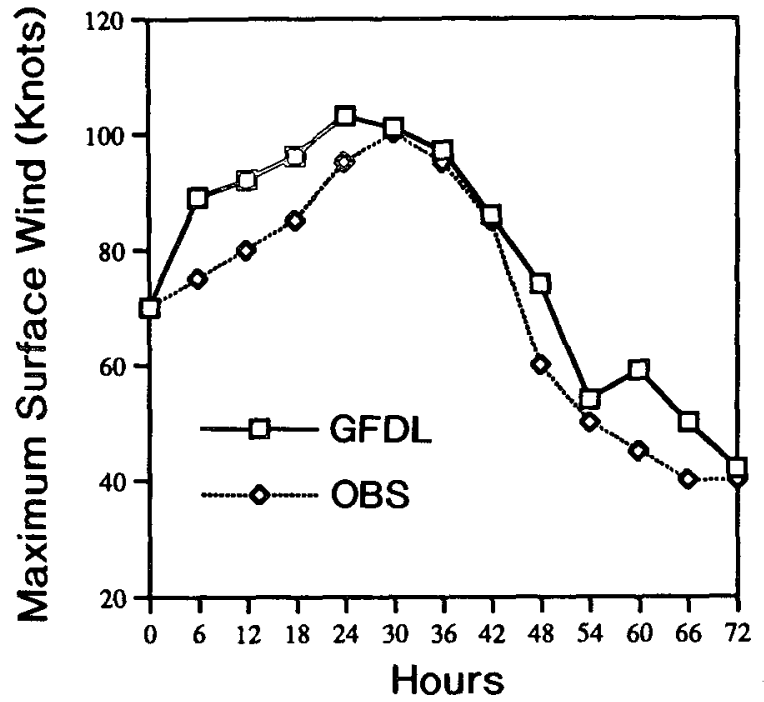

Fig. 2. Hurricane Bob's maximum surface wind speed from the GFDL hurricane model (solid) and observation (dashed) from 0000 UTC 18 to 0000 UTC 21 August 1991.

the presence of Hurricane Gloria (1985), as a means to evaluate Gloria's impact on its environment. Here we focus on investigation of the evolution of the PV perturbation field, where the PV perturbation (or anomaly) is identified as the difference of PV between the hurricane and nonhurricane simulations. To evaluate the extent to which the hurricane affects its environment, the time evolution of the azimuthal average of PV perturbations is shown in Fig. 3. The initial PV perturbation (Fig. 3a) is concentrated within the inner $2^{\circ}$ of the storm center. A small area of negative upperPV perturbation is spun up during the initialization process when the flow field is forced toward the observed values. As time passes, the spreading of the upper negative PV perturbation is clear, primarily due to the hurricane outflow advection (see Fig. 5). The azimuthal average of the negative upper-level PV perturbation covers an area of $5^{\circ}$ from the storm center at hour 12 (Fig. 3b) to $8^{\circ}$ at hour 24 (Fig. 3c), then expands beyond $10^{\circ}$ at hour 36 (Fig. 3d). At later times of the integration (Figs. $3 \mathrm{e}-\mathrm{g}$ ), a slightly downward extension of the negative upper-level PV perturbation is also found.

In the lower troposphere, the magnitude of Bob's positive PV anomaly increases in the first $36 \mathrm{~h}$ that Bob is intensifying. However, it is mainly confined in the inner storm region. This result indicates that, as far as PV is concerned, the areal extent of the hurricane's influence on its environment is far more extensive in the upper troposphere than in the lower troposphere. This is consistent with RK's findings for the temperature and wind fields. After $60 \mathrm{~h}$ of integration, as the storm dissipates over land and is tilted by a mean southwesterly to westerly vertical shear, the PV structure 

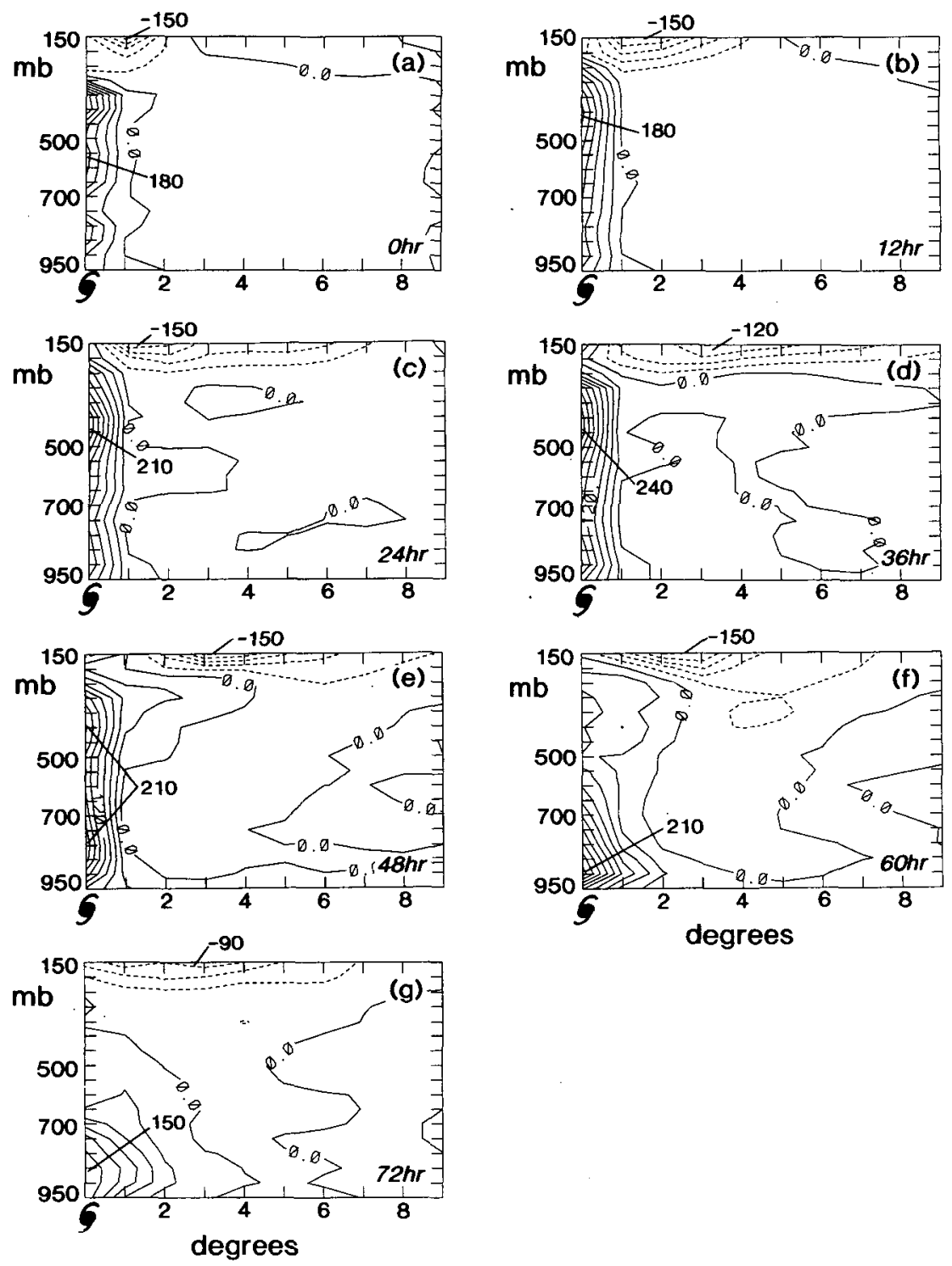

FIg. 3. The radius pressure cross section of the azimuthal average of the 72-h forecast of the potential vorticity perturbation (defined as the difference between the hurricane and nonhurricane simulation), starting from 0000 UTC 18 August 1991 at 12-h intervals. Shown are (a) 0000 UTC 18 August, (b) 1200 UTC 18 August, (c) 0000 UTC 19 August, (d) 1200 UTC 19 August, (e) 0000 UTC 20 August, (f) 1200 UTC 20 August, and (g) 0000 UTC 21 August. Values are represented in units of $0.01 \mathrm{PVU}$ (potential vorticity unit, $10^{-6} \mathrm{~m}^{2} \mathrm{~s}^{-1} \mathrm{~K} \mathrm{~kg}^{-1}$ ) with a contour interval of 0.3 PVU. Positive (negative) values are represented by solid (dashed) lines. The hurricane center is indicated by the hurricane symbol.

becomes incoherent in the vertical direction, and the concentrated PV anomaly becomes diluted (Figs. 3f and $3 \mathrm{~g}$ ).

Because Bob has experienced an increasing mean westerly vertical wind shear during the integration period, to illustrate the asymmetric distribution of the PV perturbation fields, the time evolution of the east-west cross section of the PV perturbation (through the storm center) is shown in Fig. 4. Initially, the distribution of
PV perturbation is symmetric (Fig. 4a) in the zonal direction relative to the storm center. However, as time passes (Figs. 4b-e), more negative upper PV perturbation appears toward the east (downshear side). At hour 60 (Fig. 4f), as the storm is embedded in an environment with strong westerly shear, the whole baroclinic vortex is tilted. The negative upper PV perturbation on the downshear side extends downward and becomes more distinct with a maximum value of 5 

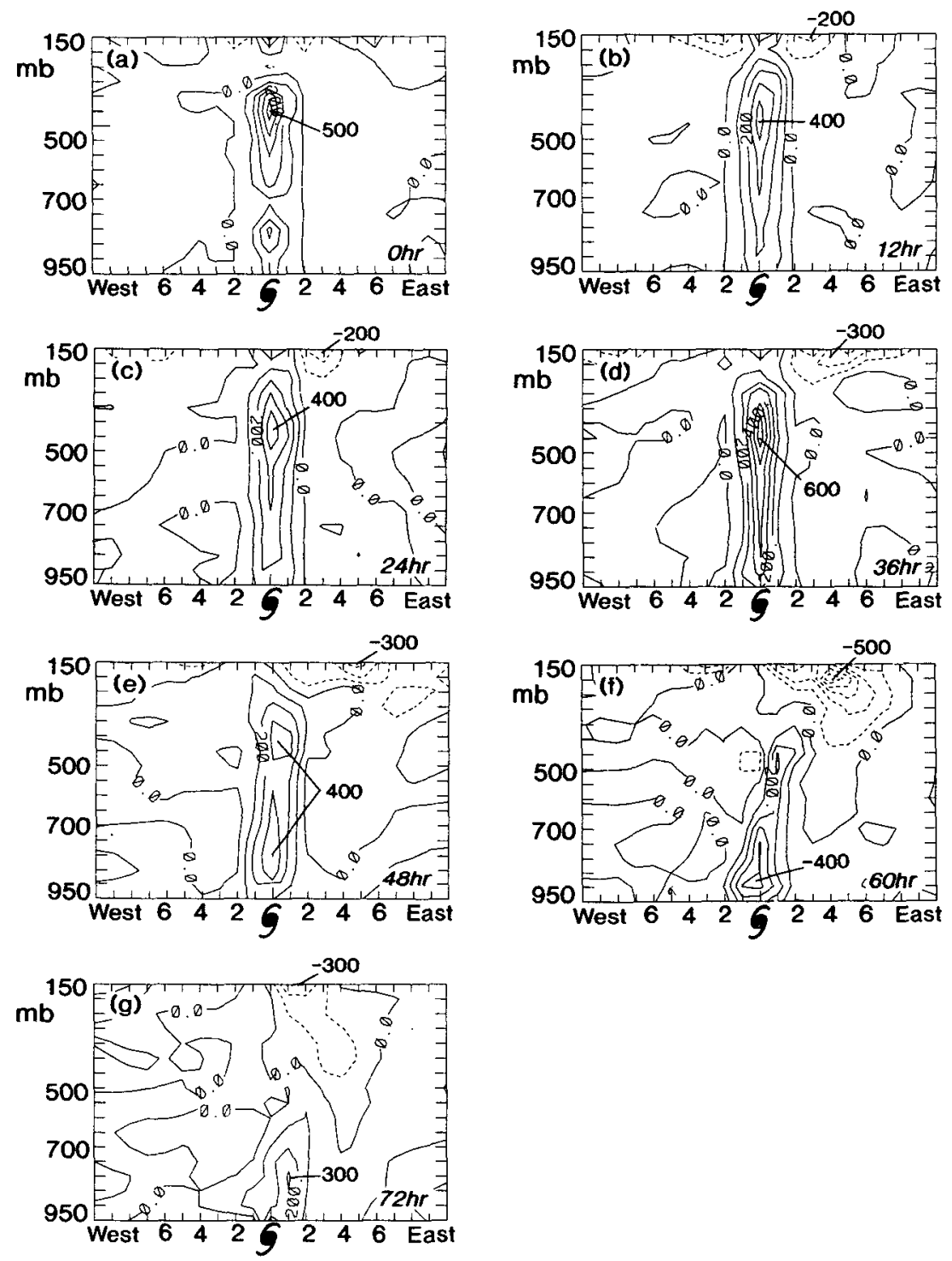

Fig. 4. The east-west cross section of the $72-\mathrm{h}$ forecast of potential vorticity perturbation, starting from 0000 UTC 18 August 1991 at 12-h intervals. Shown are (a) 0000 UTC 18 August, (b) 1200 UTC 18 August, (c) 0000 UTC 19 August, (d) 1200 UTC 19 August, (e) 0000 UTC 20 August, (f) 1200 UTC 20 August, and (g) 0000 UTC 21 August. Values are represented in units of 0.01 PVU with a contour interval of 1 PVU. Positive (negative) values are represented by solid (dashed) lines. The hurricane center is indicated by the hurricane symbol.

PVU (potential vorticity unit, $10^{-6} \mathrm{~m}^{2} \mathrm{~s}^{-1} \mathrm{~K} \mathrm{~kg}^{-1}$ ), while the vertical extent of the lower positive PV shrinks. This downshear expansion of the negative upper-level PV perturbation resembles the picture portrayed in WEM93.

As was indicated in WEM95a,b an alternative and more concise approach to having a better understanding of the evolution of the PV perturbation in the upper troposphere is to look at the so-called dynamical tropopause potential temperature (hereafter DTPT) field at the 1.5-PVU surface. Figure 5a shows both the wind and potential temperature fields at the dynamical tropopause. Initially, there is a tiny closed area of a cold 355-K DTPT region surrounded by a warm $360-\mathrm{K}$ DTPT annulus above Bob. The former cold area represents the vertical extension of Bob's cyclonic vorticity, and the latter warm annulus is equivalent to a patch of low-PV air in the upper troposphere. The anticyclonic outflow is also obvious in Fig. 5a.

To further aid visualization of the effect of the storm's outflow on the evolution of the DTPT contour, Fig. 5b shows only the component of wind field that is 


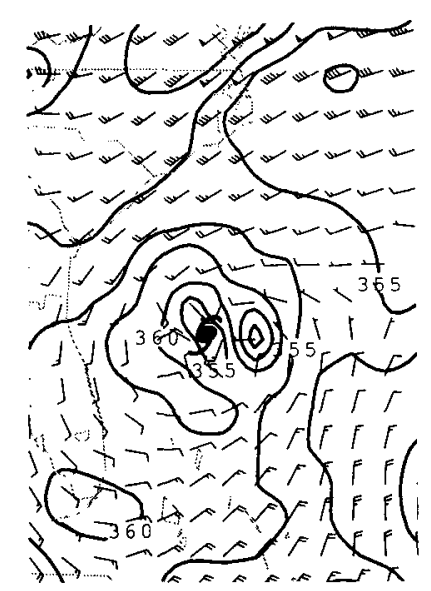

(a) 1.5 PVU SFC THETA \& WIND FCSTOO $910818 / 00$

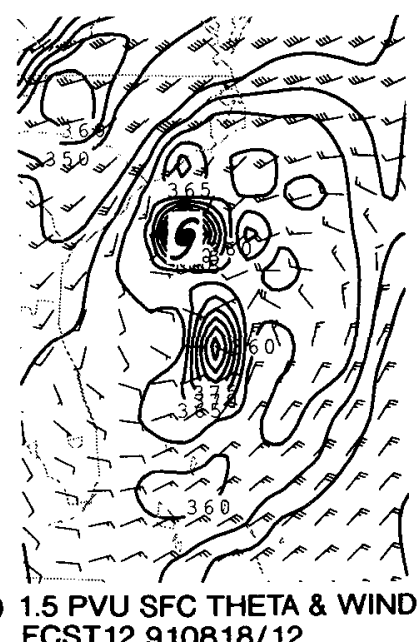

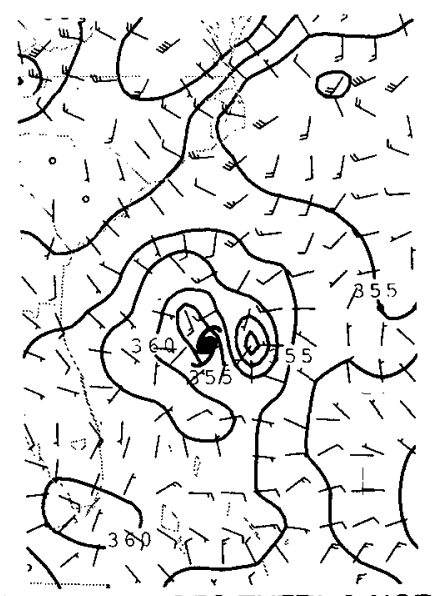

(b) 1.5 PVU SFC THETA \& NORM-WIND FCSTOO $910818 / 00$

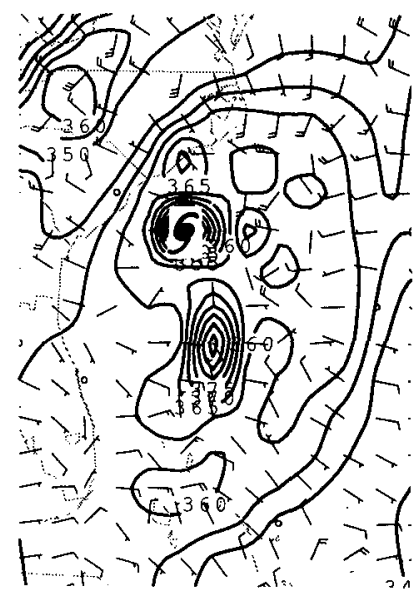

(d) 1.5 PVU SFC THETA \& NORM-WIND FCST $12910818 / 12$

FIG. 5. The tropopause potential temperature and wind fields (on the 1.5 PVU surface) at (a) 0000 UTC 18 August and (c) 1200 UTC 18 (12-h forecast) August 1991. Panels (b) and (d) are identical to (a) and (c) except that the component of wind in the direction perpendicular to the potential temperature contour is plotted. The contour interval is $5 \mathrm{~K}$. One long barb indicates $10 \mathrm{kt}\left[5 \mathrm{~m} \mathrm{~s}^{-1}\left(8-12 \mathrm{kt} ; 4-6 \mathrm{~m} \mathrm{~s}^{-1}\right)\right]$; one short barb indicates $5 \mathrm{kt}\left[2.5 \mathrm{~m} \mathrm{~s}^{-1}(3-7 \mathrm{kt} ; 1.5-\right.$ $\left.3.5 \mathrm{~m} \mathrm{~s}^{-1}\right)$ ]; no barb indicates winds less than $3 \mathrm{kt}\left(1.5 \mathrm{~m} \mathrm{~s}^{-1}\right)$; and " 0 "' indicates no wind. The model's hurricane positions are indicated by the hurricane symbol.

parallel to the direction of the potential temperature gradient. It is clear that the divergent outflow plays a major role in advecting the whole high potential temperature air outward. Twelve hours later (Fig. $5 \mathrm{c}$ ), the cold region above Bob is still confined in a small area above Bob, while the warm $360-\mathrm{K}$ contour has covered a much broader area, and a local warm maximum is located to the south-southeast of Bob's center. The deformation of the $360-\mathrm{K}$ contour to the south of the hurricane appears to be the result of the self-advection process. At hour 12, the component of the wind normal to the DTPT contour from Fig. 5d also indicates that the outflow is effectively advecting the high potential tem- perature air outward. This is in agreement with WEM93's idealized model where they assumed that a divergent potential flow, emanating from a source that coincides with the storm position, carries the lens of low-PV air in the upper troposphere outward.

At hour 24 (Fig. 6a), except for two small local cold DTPT areas near Bob's center, several high DTP'T centers surrounding Bob exist, and the areal extent of the $360-\mathrm{K}$ contour becomes even larger. But at this time, Bob is moving toward higher latitude where the flow field is dominated by a strong southwesterly wind associated with an approaching upper-tropospheric trough over the central United States. From then on, 


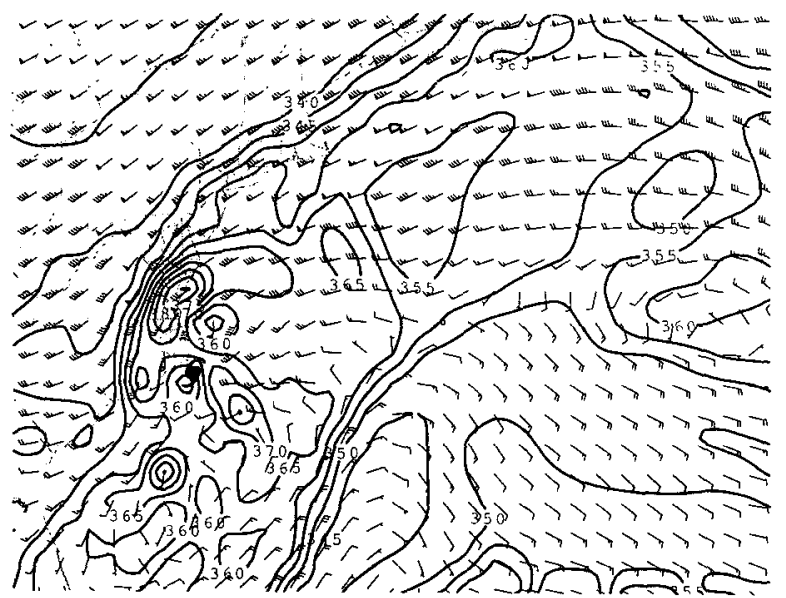

(a) 1.5 PVU SFC THETA \& WIND FCST24 910819/00

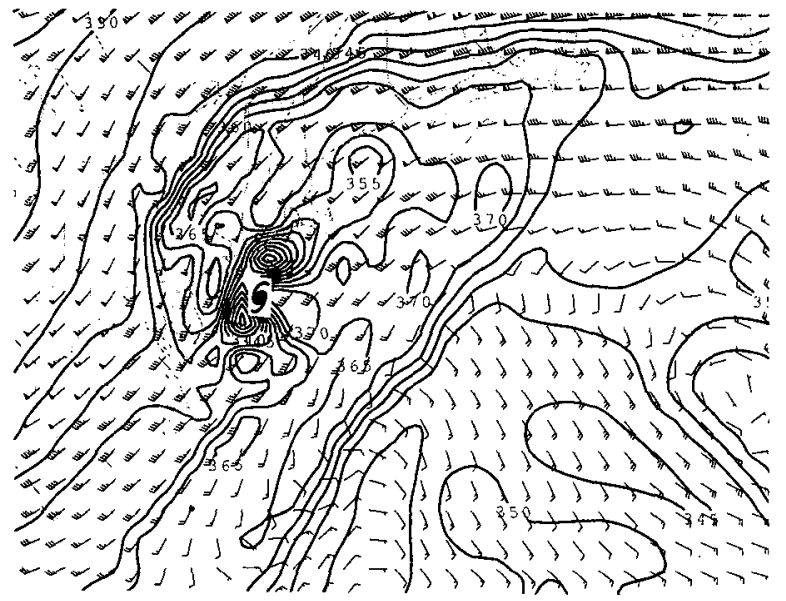

(b) 1.5 PVU SFC THETA \& WIND FCST 36 910819/12

Fig. 6. The tropopause potential temperature and wind fields (on the 1.5-PVU surface) at (a) 0000 UTC 19 August (24-h forecast) and (b) 1200 UTC 19 August (36-h forecast) 1991. The contour interval is $5 \mathrm{~K}$. The wind barb is as in Fig. 5. The model's hurricane positions are indicated by the hurricane symbol.

most of the high potential temperature air is advected to the downshear side. As can be seen from the potential temperature distribution at hour 36 (Fig. 6b), even though two local cold DTPT centers exist to the northnorthwest and south-southwest of Bob, a great portion of the $360-\mathrm{K}$ contour has been advected northeastward relative to the hurricane center. In other words, most of the warm DTPT air has been advected toward the downshear side. Here the effect from the divergent hurricane outflow has been overwhelmed by the strong advection due to the synoptic-scale background flow. This is also in agreement with WEM94's finding where they showed that the upper PV anomaly in the numerical experiments may either expand and rotate around the storm or propagate quickly to the downshear side, depending on the relative magnitude of the divergent potential flow and the background vertical shear.
Figure 7 shows the time evolution of the potential temperature perturbation on the dynamical tropopause. Initially (Fig. 7a), there is an area of $5-\mathrm{K}$ warm anomaly surrounding Bob. Twelve hours later (Fig. 7b), the 5-K warm anomaly has expanded in area and has experienced some deformation, which looks like the classic picture of the vortex's self-advection. At hour 24 (Fig. 7c), the area of 5-K anomaly increases slightly, a small warm tongue extending to the south due to the advection by the anticyclonic outflow. At hour 36 (Fig. 7d), more anomaly has been advected to the downshear (northeast) side. At hour 48 (Fig. 7e), the whole warm DTPT perturbation is primarily advected to the east by the upper westerly shear. This feature is very similar to the observed DTPT anomaly map in WEM95a (cf. their Fig. 9f). At hour 60 (Fig. 7f), more warm anomaly has been advected to the downshear side. Meanwhile, some warm anomaly has bent toward the southeast of Bob, possibly advected by its own associated anticyclonic flow. This pattern also bears some similarity to the upper-layer PV feature in the model result of WEM93 (cf. their Fig. 5). Near the end of the integration (Fig. $7 \mathrm{~g}$ ), more filamentation forms and the warm anomaly evolves into a much more complicated pattern.

Besides the expansion in area of the warm potential temperature anomaly, another important feature that should be noted in Fig. 7 is the strengthening of the warm potential temperature anomaly on the dynamical tropopause with time. The magnitude of the warm anomaly increases from $5 \mathrm{~K}$ initially (Fig. 7a) to $10 \mathrm{~K}$ at hour 12 (Fig. $7 \mathrm{~b}$ ), $15 \mathrm{~K}$ at hour 24 (Fig. 7c), and $20 \mathrm{~K}$ at hour 36 (Fig. 7d). The maximum potential temperature anomaly of $25 \mathrm{~K}$ is found at hour 60 (Fig. 7f). Clearly, the increase of the DTPT perturbation must have resulted from some nonconservative processes. To distinguish these processes in a quantitative manner, the PV budget is analyzed in detail. The local changes of PV due to the horizontal advection, vertical advection, and the effect of heating and friction are examined individually.

\section{c. PV budget analysis}

To be consistent with the calculation in WEM95, we formulate our calculation of PV budget on the $\pi$ coordinate [Exner function: $\left.\pi=C_{p}\left(p / p_{0}\right)^{\kappa}\right]$. The approximate definition of Ertel's PV in $\pi$ coordinates is

$$
q=-\frac{g \kappa \pi}{p}\left(\eta \frac{\partial \theta}{\partial \pi}-\frac{1}{a \cos \varphi} \frac{\partial v}{\partial \pi} \frac{\partial \theta}{\partial \lambda}+\frac{1}{a} \frac{\partial u}{\partial \pi} \frac{\partial \theta}{\partial \varphi}\right),
$$

where $\kappa=R_{d} / C_{p}, p$ is the pressure, $\eta$ is the vertical component of absolute vorticity, and $\theta$ is the potential temperature. The PV budget is 

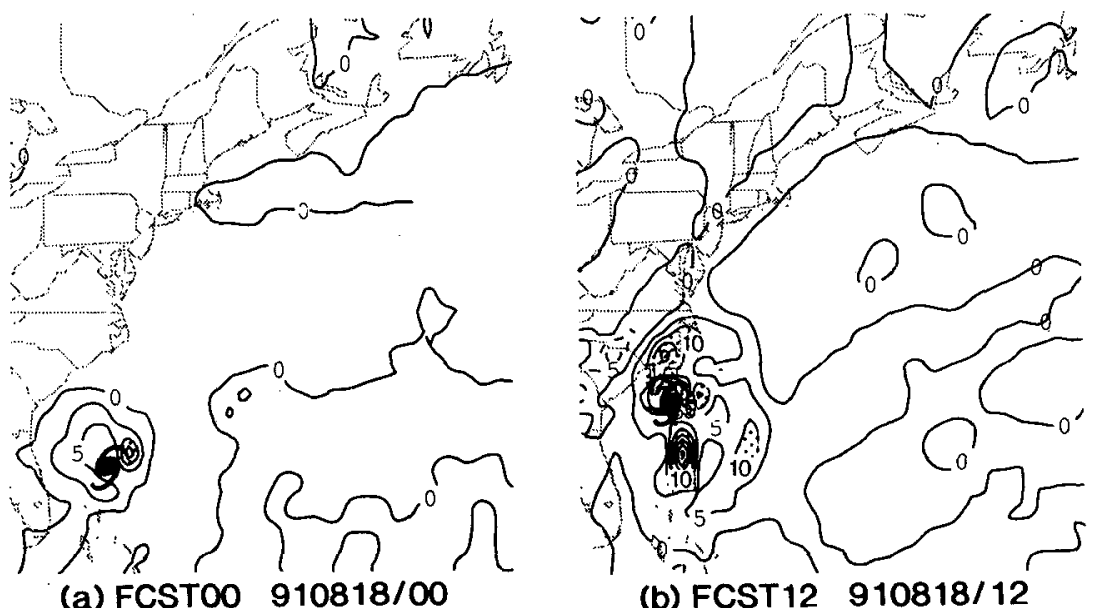

(b) FCST $12 \quad 910818 / 12$

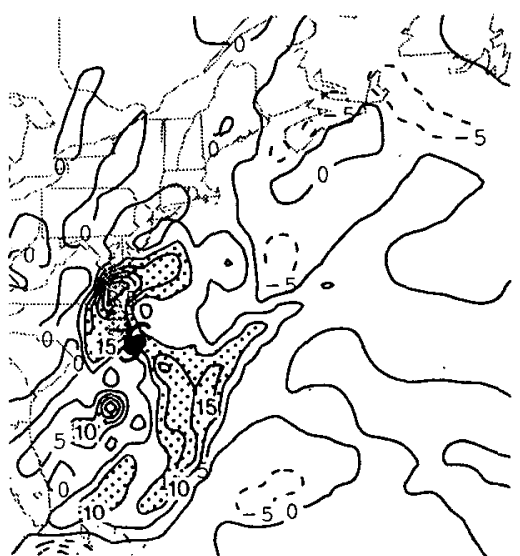

(c) FCST24 910819/00

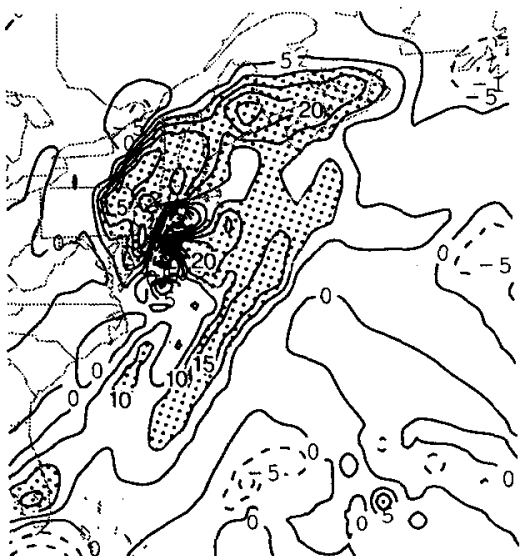

(d) FCST36 $910819 / 12$

FIG. 7. The time evolution of the tropopause potential temperature perturbation fields (on the 1.5-PVU surface) from 0000 UTC 18 August to 0000 UTC 21 August at 12-h intervals. Shown are (a) 0000 UTC 18 August, (b) 1200 UTC 18 August, (c) 0000 UTC 19 August, (d) 1200 UTC 19 August, (e) 0000 UTC 20 August, (f) 1200 UTC 20 August, and (g) 0000 UTC 21 August. The contour interval is $5 \mathrm{~K}$. All positive (negative) values are represented by solid (dashed) lines. The model's hurricane positions are indicated by the hurricane symbol. All values equal to or larger than $10 \mathrm{~K}$ are shaded.

$$
\begin{aligned}
\frac{\partial q}{\partial t}=-v_{h} \cdot \nabla q- & \omega^{*} \frac{\partial q}{\partial \pi} \\
& -\frac{g \kappa \pi}{p}\left[\eta \cdot \nabla\left(\frac{d \theta}{d t}\right)+\nabla \theta \cdot \nabla \times F\right],
\end{aligned}
$$

where $\omega^{*} \equiv d \pi / d t$ and the terms on the right represent the local change of PV due to the horizontal advection, vertical advection, and the effect from diabatic heating $(d \theta / d t$, including the condensational and radiative heating) and friction $(F)$, respectively. It should be noted that the analysis may reduce numerical accuracy due to the interpolation from the model's sigma coordinate to the $\pi$ coordinate. Also, numerical results derived from coarse-resolution analysis of models in non- conservation form may not exactly agree with theoretical conclusions on the potential vorticity budget (Haynes and McIntyre 1987). However, as will be shown later, the effect from both the vertical motion and condensational heating is excessively large in the inner part of Bob. It is anticipated that the effiects due to the above terms are predominant in the PV budget analysis in any of the $\pi$, sigma, or $\theta$ coordinates. It can be assumed that a major feature of the PV budget is captured from this analysis.

The zonal cross sections for the different terms of the PV budget analysis at forecast hour 12 are shown in Fig. 8. The cross section of PV (Fig. 8a) clearly shows that the high PV air is concentrated within the inner $2^{\circ}$ of the storm center. It has a local maximum 


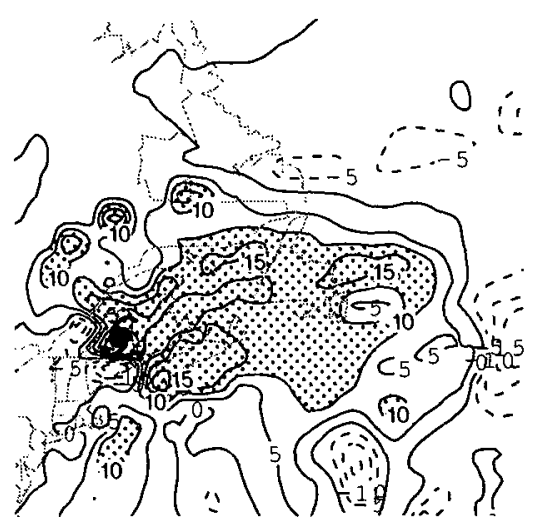

(e) FCST48 $910820 / 00$

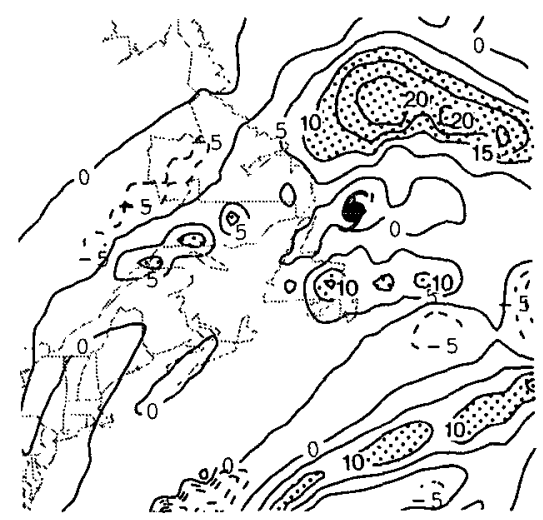

(g) FCST72 $910821 / 00$

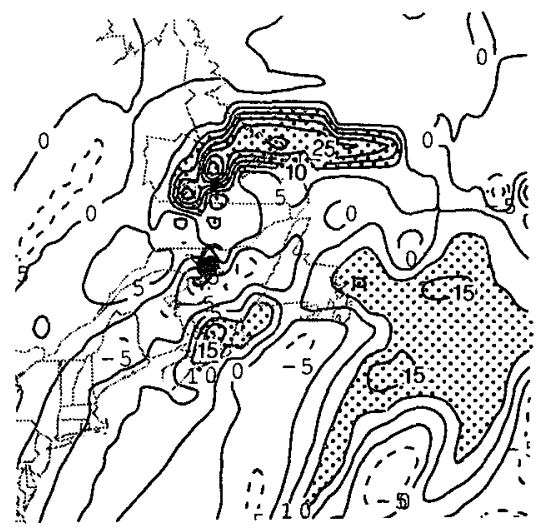

(f) FCST60 $910820 / 12$

FIG. 7. (Continued)

value of 4 PVU at about $450 \mathrm{mb}$. Also, the local PV maximum extends vertically up to about $200 \mathrm{mb}$. The vertical motion field (Fig. 8b) indicates that strong updrafts exist near the hurricane center, with a maximum vertical velocity of about $-5 \mathrm{~Pa} \mathrm{~s}^{-1}$ (about $0.5 \mathrm{~m} \mathrm{~s}^{-1}$; the actual maximum upward motion is stronger when calculated from finer-resolution grids, e.g., $1 / 3^{\circ}$ or $1 / 6^{\circ}$ resolution). There is a very weak positive (negative) horizontal PV advection to the downshear side (upshear side) of Bob through the troposphere (not shown). This horizontal PV advection is much weaker compared to those terms associated with the vertical advection and the condensational heating. The local change of the PV tendency due to the vertical advection (Fig. 8c) is strongest at Bob's center. Physically, the strong updraft near the hurricane center carries the high PV air from the lower troposphere to the upper troposphere, thus producing a maximum positive PV tendency of 1.2 PVU $(12-\mathrm{h})^{-1}$ at about the $300-\mathrm{mb}$ level.

The distribution of condensational heating (Fig. 8d) is consistent with that of upward motion (Fig. 8b). The result suggests that the condensation occurs mainly at the resolvable scale in the model since we have also conducted simulations with the cumulus parameteriza- tion turned off and have produced similar heating profiles. (No absolute convective instability occurs in these no-convective-adjustment simulations, probably due to the effect of strong vertical mixing within the vortex.) The maximum condensational heating (about $22 \mathrm{~K} \mathrm{~h}^{-1}$ ) occurs near the storm center at about 400 $\mathrm{mb}$. This condensational heating profile causes the redistribution of PV in the vertical direction (Fig. 8e) by creating a negative PV anomaly [at a rate of about -2 PVU $\left.(12 \mathrm{~h})^{-1}\right]$ in the upper troposphere and positive PV anomaly in the lower troposphere.

Our calculations also show that the local change of PV due to other nonconservative processes (including both the radiative heating and friction) is more than an order of magnitude smaller than that due to the condensational heating. In other words, condensational heating is the primary nonconservative factor affecting Bob's PV evolution. The local change of PV due to all terms, including the total advection, heating, and friction (Fig. 8f) indicates that there exists a source of PV in the middle to lower troposphere and a sink of PV in the upper troposphere at Bob's center. Similar results are also found at other forecast times (not shown). 


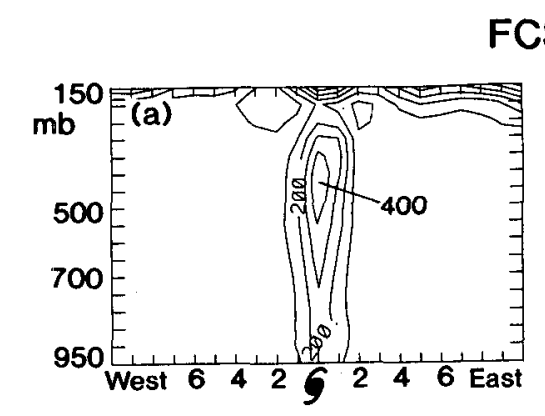

FCST 12HR
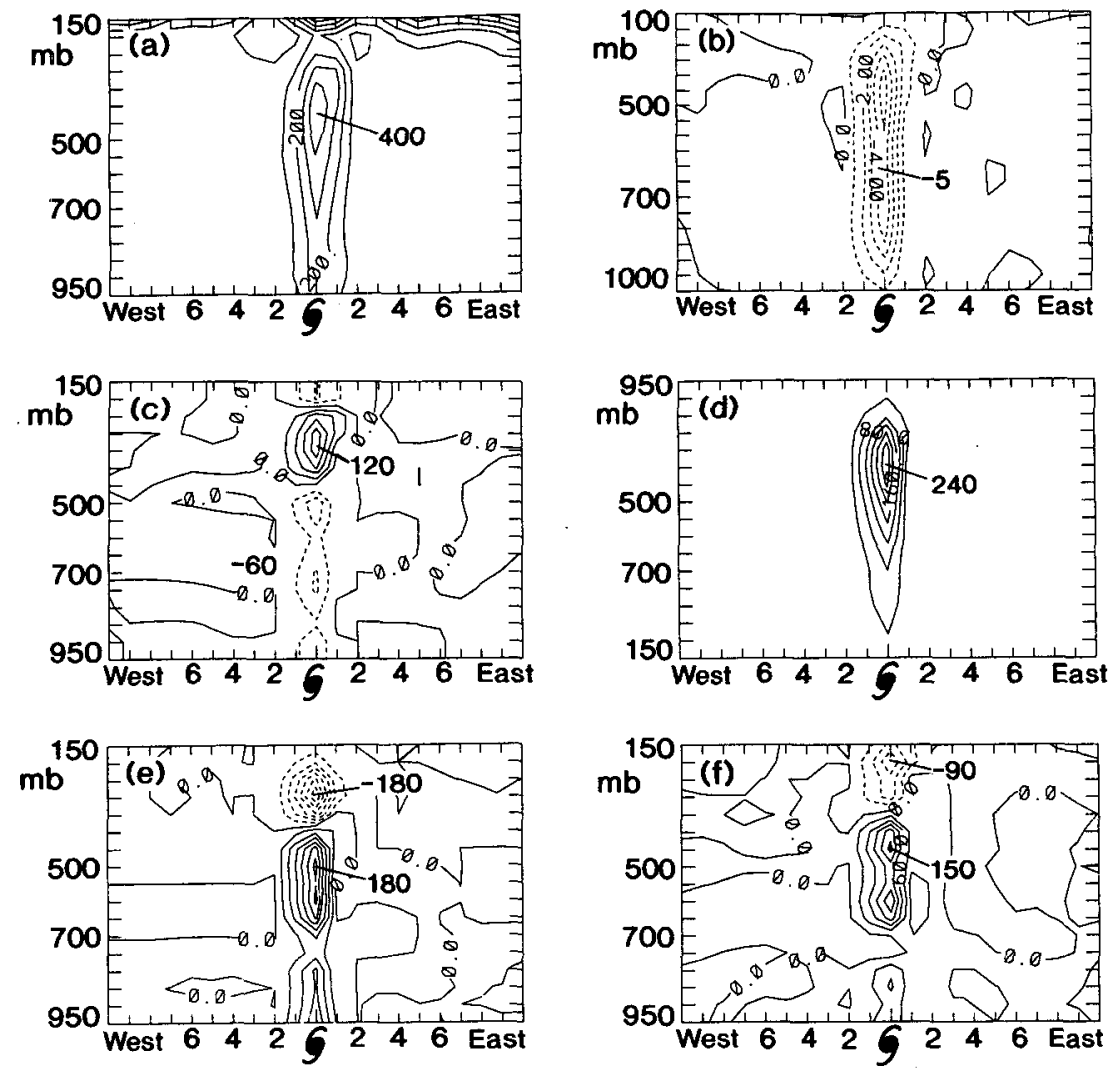

FIG. 8. The east-west cross section of the 12-h forecast at 0000 UTC 18 August for (a) potential vorticity (units of $0.01 \mathrm{PVU}$ with a contour interval of $1 \mathrm{PVU}$ ), (b) vertical motion $\left(\omega\right.$, contour interval of $\left.1 \mathrm{~Pa} \mathrm{~s}^{-1}\right)$, the change of potential vorticity due to (c) vertical advection, (e) condensational heating, and (f) total effect of advection, heating, and friction (units of 0.01 PVU/12 h with a contour interval of 0.3 PVU/12 h). All positive (negative) values are represented by solid (dashed) lines. (d) The condensational heating rate (units of $\mathrm{K} / 12 \mathrm{~h}$ with a contour interval of $40 \mathrm{~K} / 12 \mathrm{~h}$ ). Positive (negative) values are represented by solid (dashed) lines. The hurricane center is indicated by the hurricane symbol.

The PV budget analysis clearly demonstrates that vertical advection and the effect of condensational heating are the two dominant processes causing the local change of PV for Bob. One interesting point is that the strong updraft at the storm center plays dual roles: Dynamically, it transports high PV air upward, but thermodynamically it leads to the condensation of the moist air and therefore causes the redistribution of PV. These two effects tend to oppose each other. However, in our study, we find that the heating effect is dominant. This result indicates the importance of the diabatic heating in influencing the evolution of the storm's structure. This will later feed back to affect storm movement, as will be shown in section $4 \mathrm{~d}$.

Over all, the above findings can be summarized as follows: The generation of the negative PV in the upper troposphere is mainly diabatically generated due to the strong condensational heating near the hurricane center. From the Lagrangian point of view, the air parcel moving upward near the storm center tends to lose PV along its way above the level of maximum diabatic heating. As this low PV air reaches the outflow layer, it is advected away from the storm by both the divergent outflow (as indicated from Fig. 5) and the uppertropospheric background mean wind. This process agrees well with the scenario presented in WEM93.

\section{d. PV diagnostics}

In this section, the PV diagnostics, as desciibed in WEM95a, are performed to evaluate the contribution of the upper PV anomaly to the storm's advecting flow. As a first step to demonstrate that such a PV method is valid, we compare the inverted balanced wind field and the actual wind field from the model (same procedure as discussed in section 4a of WEM95a). In the early period of the integration, in the lower levels there exists a strong southerly steering over Bob (not shown), ap- 
parently due to a combination of the flow associated with the trough over the central United States and the high pressure system over the Atlantic. This feature is well presented in both the model and the balanced wind fields. The two flow fields agree well over most of the integration domain except near the boundary and the storm center, where Bob's cyclonic circulation is underestimated in the balanced flow. At upper levels, the meandering westerlies across the United States and the Atlantic are well captured in both the model and balanced winds. Except for points near the boundary, their difference is less than $5 \%$ of the magnitude of the flow itself. Therefore, we regard the nonlinear balanced flow, especially for that in the upper troposphere, as a good approximation to the flow field simulated from the GFDL hurricane model and our PV diagnostics as a justified approach for evaluating the effect of the upper-tropospheric PV anomaly on the hurricane's steering flow.

To determine whether the flow associated with the generated PV perturbation in the outflow area can indeed effectively steer the storm, a piecewise PV inversion is performed (as in WEM95a) on upper PV perturbations. The balanced flow associated with the upper PV perturbation in the outflow region (between 300 and $100 \mathrm{mb}$ ) is calculated, and interpolation is made of the flow at the vortex center to evaluate their influences on storm movement. Figure 9 shows an example of the flow field associated with the PV perturbation in the outflow region at hour 48 , as indicated by the $1.5-$ PVU surface potential temperature map (Fig. 7e). At $150 \mathrm{mb}$ (Fig. 9a), the anticyclonic circulation associated with the negative PV anomaly is extensive. The flow associated with the upper PV anomaly also penetrates downward to the lower troposphere (Fig. 9b). However, the anticyclonic circulation at $700 \mathrm{mb}$ (Fig. $9 \mathrm{~b}$ ) is centered to the north of Bob, while the $150-\mathrm{mb}$ anticyclonic circulation (Fig. 9a) is centered to the east. This tilt is resulted from the incoherent vertical distribution of PV anomalies in the outflow region, where the negative PV anomalies are mainly located to the north of Bob at 250- and 300-mb levels, but more negative PV anomalies exist to the east at higher levels. Nevertheless, the $850-300 \mathrm{mb}$ vertically averaged balanced flow (associated with the outflow PV perturbation) at Bob's center contributes in advecting Bob northwestward at a speed of about $3 \mathrm{~m} \mathrm{~s}^{-1}$.

The 850-300 mb vertically averaged balanced flows at the vortex center, associated with different PV fields from forecast hours 12 to 48 , are shown as hodographs in Fig. 10, where " $U$ ' represents the flow associated with the PV perturbation in the outflow region between 300- and 100-mb levels, "L" represents the flow associated with the PV perturbation between the 1000and $350-\mathrm{mb}$ levels (note that $\mathrm{L}$ here is equivalent to " $L E$ "' in WEM95a, where the PV anomaly of the storm itself has been excluded to avoid the contamination of the hurricane's strong azimuthal circulation resulted from the sensitivity of the hurricane center for interpolation ), "Mean" indicates the flow from the nonhurricane simulation at the presumed storm center, and "TC' represents the actual model vortex motion.

Figure 10 shows that the summation of the flow associated with all PV fields (Mean $+U+L$ ) is a good approximation to Bob's actual track. This again confirms the validity of the PV diagnostics. It appears that the advecting flow associated with Mean is heading to the right of the actual vortex motion in the model. At hours 12 and 24, this difference can be largely compensated by the northwestward flow associated with $\mathrm{L}$, which is essentially induced by the asymmetric $\beta$ gyres (see Fig. 12). At hours 36 and 48, the advecting flow associated with L shifts toward the west and southwest. Meanwhile, the contribution from the upper perturbed PV field (U) becomes more significant in time.

The effect from the upper PV anomaly (U) can be interpreted as the following. Initially (Fig. 10a), as the upper negative PV anomaly is small in its horizontal scale and is right above Bob, its influence on the storm movement is almost negligible. However, as the upper PV anomaly is spread out toward the northeast side due to the southwesterly shear flow, its contribution in advecting Bob northwestward becomes stronger-from a northwestward advection of $1 \mathrm{~m} \mathrm{~s}^{-1}$ at hour 24 (Fig. $10 \mathrm{~b}$ ), to $2 \mathrm{~m} \mathrm{~s}^{-1}$ at hour 36 (Fig. 10c), and finally 3 $\mathrm{m} \mathrm{s}^{-1}$ at hour 48 (Fig. 10d). When only the negative upper PV anomaly located above and to the downshear side of Bob is inverted, its associated flow has even stronger steering effect (not shown). Combining the result of this PV diagnostics with the previous finding that the upper negative PV anomaly is mainly generated by the diabatic heating process in Bob, the effect proposed in WEM93 is demonstrated.

In addition to Bob, we have also studied two other cases, namely Andrew (1992) and Gilbert (1988), which are more typical storms over the Tropics. In these cases, the sink of PV due to the condensational heating effect above the storm is also found. However, due to the weaker and time-varying environmental wind shear and its direction (so there is no obviously consistent downshear tilt of the negative upper PV anomaly toward any specific direction) and the less efficient coupling between the lower vortex and upper negative PV anomaly in the lower-latitude region, the effect from the upper-level PV anomaly on the advection of both Andrew and Gilbert is weaker (about 0.5$1 \mathrm{~m} \mathrm{~s}^{-1}$ ). Both Andrew's and Gilbert's steering currents are dominated by the flows associated with Mean and L. Even though the results from our study of Bob agree well with the theory presented in WEM93, WEM94, and WEM95a,b, the different findings from two other cases suggest the case dependence of the effect of the upper-level PV perturbation on storm movement. It is speculated that Bob is embedded in a more baroclinic environment to allow the interaction mech- 


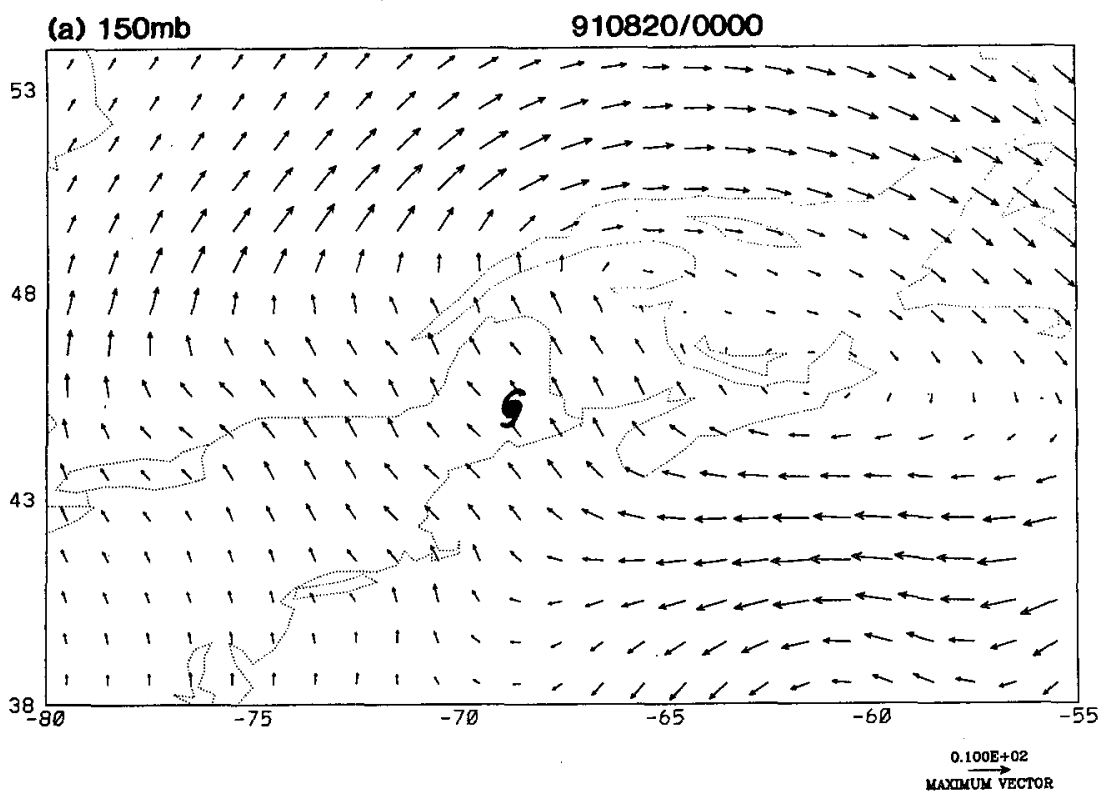

(b) $700 \mathrm{mb}$

$910820 / 0000$

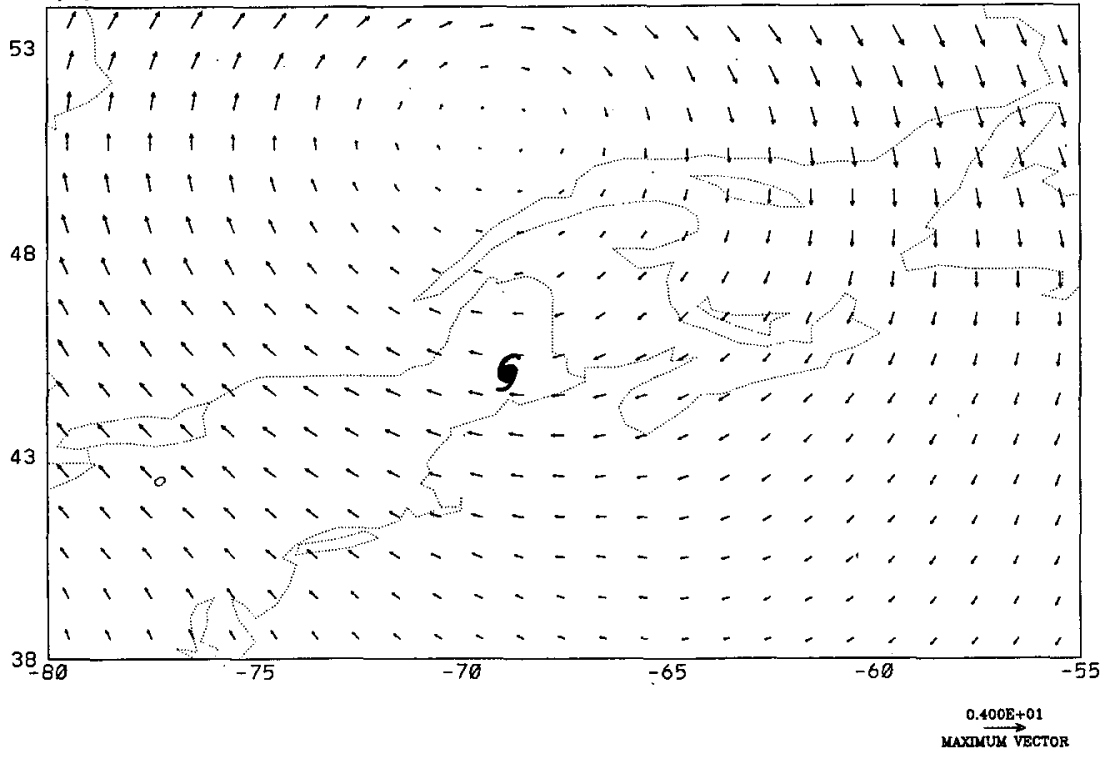

FIG. 9. Balanced wind fields associated with the potential vorticity perturbation (U) between 300 and $100 \mathrm{mb}$ at 0000 UTC 20 August for (a) 150 and (b) $700 \mathrm{mb}$. The model's hurricane position is indicated by the hurricane symbol.

anism described in WEM93 to play a more important role in steering Bob.

\section{e. Beta effect}

As mentioned in section 3, during the initialization process an asymmetric beta-gyre structure is added. In Bob's case, as shown in Fig. 11, the initial advecting flow from the bogused dipole gyres is about $1.6 \mathrm{~m} \mathrm{~s}^{-1}$ through Bob's center. During the model integration, due to the strong cyclonic circulation around the hurricane center, separating the flow associated vith the beta gyres from that of the storm becomes difficult. Here we have adopted three different methods to look for the $\beta$ effect. The first approach is a more or less traditional method, that is, to remove the axisyrnmetric flow field relative to the storm center, then to examine the asymmetric component of the perturbation wind and PV field, supposing that the $\beta$ gyres should be preserved in this analysis. Although there is a clear wave- 


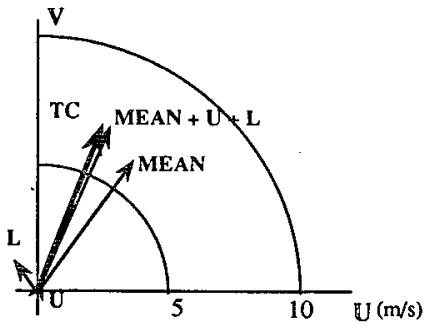

$910818 / 1200$

(a) FCST $12 \mathrm{HR}$

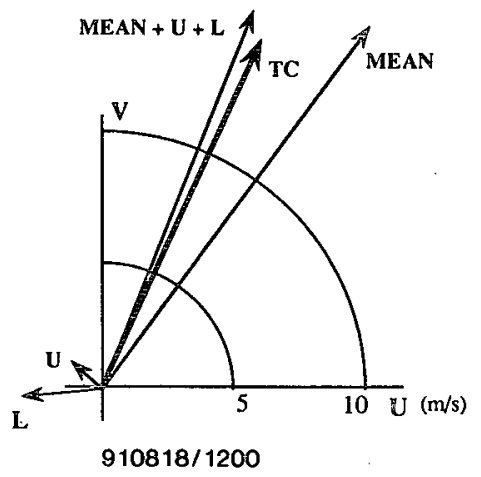

(c) FCST 36HR

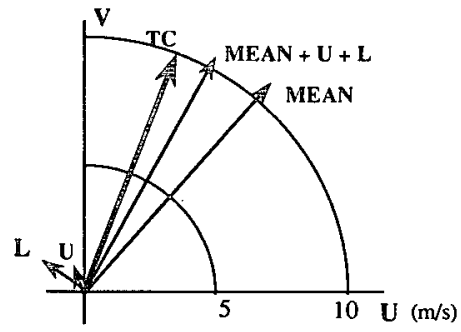

$910819 / 0000$

(b) FCST $24 H R$

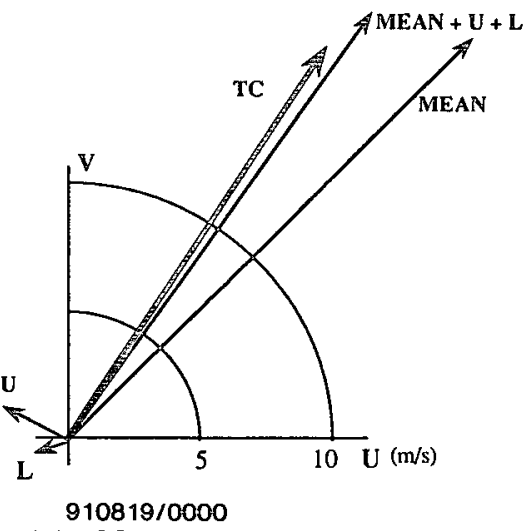

(d) FCST $48 \mathrm{HR}$

FIG. 10. Velocity vectors of the balanced flows (interpolated to Bob's center) and Bob's direction and speed of motion from 1200 UTC 18 August (12-h forecast) to 0000 UTC 20 August (48-h forecast). Shown are (a) 1200 UTC 18 August, (b) 0000 UTC 19 August, (c) 1200 UTC 19 August, and (d) 0000 UTC 20 August. Mean, U, and L represent the 850-300$\mathrm{mb}$ pressure-averaged balanced flows associated with the background potential vorticity, balanced flows associated with the potential vorticity perturbations between 300 and $100 \mathrm{mb}$, and those between 1000 and $350 \mathrm{mb}$, respectively. TC indicates model-predicted Bob's instant motion.

number-1 asymmetric flow at the initial time (Fig. 11), the asymmetric flow and PV fields quickly become more complicated as the model integration proceeds.

The asymmetric wind and PV fields at the $700-\mathrm{mb}$ level at hours 12 and 24 are shown in Fig. 12. Near the storm center, the distribution of wind and PV fields are not realistic due to the sensitivity of the storm center in calculating the asymmetric flow. Strong and unrealistic asymmetric flow and PV fields are found near the vortex core. To avoid contamination of these strong winds and the bull's-eyes of the PV field in the diagram, in Fig. 12 only the wind vector less than 4 (or 8 ) $\mathrm{m} \mathrm{s}^{-1}$ is plotted for panel (a) [or panel (b)], and the positive PV anomaly larger than $0.5 \mathrm{PVU}$ at the storm center is not plotted. At hour 12 (Fig. 12a), two clear gyres exist a few degrees outside of the hurricane center with a broad anticyclonic flow to the northeast and a smaller cyclonic circulation to the southwest. At hour 24 (Fig. $12 \mathrm{~b}$ ), these gyres becomes even stronger. Not only the flow field, but also the PV anomaly dipole is distinct in Fig. 12. Therefore, in the first $24 \mathrm{~h}$ of the model integration, strengthening of the two counter- rotating gyres occurs. This is consistent with what the $\beta$ effect has suggested. At later times of the model integration, the flow patterns become more complicated (not shown), and no clear quasi-steady $\beta$ gyres can be found. On the other hand, for the cases of Andrew and Gilbert, the flow structure is much more complicated at all times. It appears that the background vorticity gradient may have been distorted by the ambient flow so that no simple $\beta$ gyres can be generated or maintained. Whether we are able to find $\beta$ effect is case dependent.

Instead of removing the axisymmetric flow relative to the storm center to detect the $\beta$ gyres, another approach tested is to remove the hurricane vortex by the method developed in the initialization package of the GFDL hurricane model (KBTR, see section 3) and to detect the gyres in the remaining environmental flow. Nevertheless, unlike the results from the previous method, our results (not shown) from this method do not show any conclusive beta effect from any of the three cases. One possible explanation of the inconsistency between the two methods in detecting Bob's $\beta$ gyres is that the second method may 


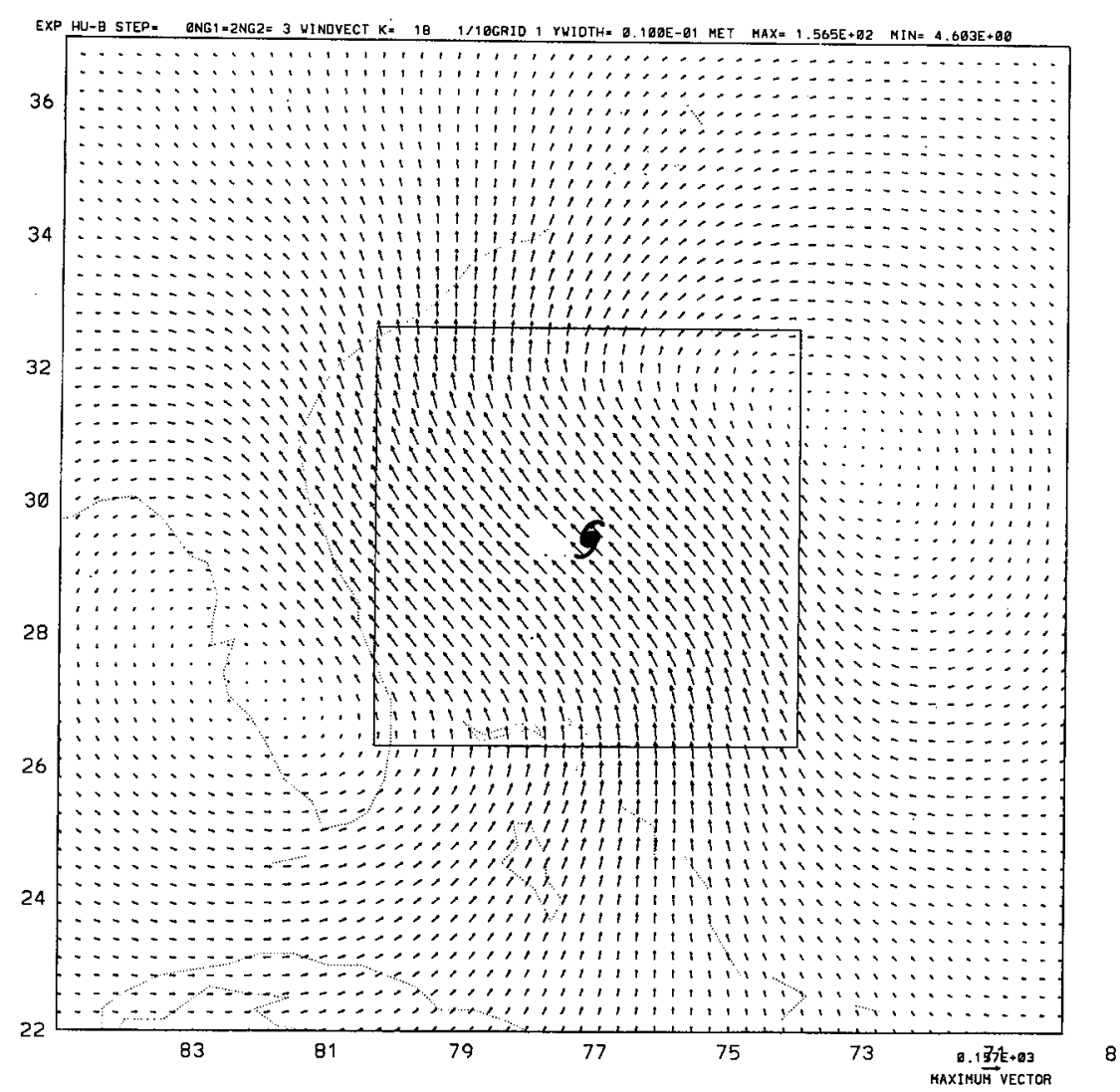

FIG. 11. Wind vectors of the initial bogused asymmetric flow field at 0000 UTC 18 August for the regions of the inner two nests of the GFDL hurricane model. The maximum wind vector is $1.57 \mathrm{~m} \mathrm{~s}^{-1}$.

have removed the storm vortex in a domain so loose that it contains the $\beta$ gyres, which is found by the first method.

We also investigate the $\beta$ effect by the method discussed in WEM95a (see their Fig. 14), that is, we use the hurricane vortex's associated balanced flow to advect the background PV field (from the nonhurricane simulation). This is a simple way to examine the hurricane's direct influence on its background flow and to compare it with the conceptual numerical model that supports the theory of the $\beta$ effect. It turns out that some high wavenumber PV gyres surrounding the hurricane vortex are found (not shown), and no clear wavenumber-1 beta gyres can be detected for all three cases. Comparing this result with the finding of $\beta$ gyres in Bob from the first method shows that the production of the gyres is nonlinear and cannot be simply interpreted from the linear vorticity advection. The nonlinear effect must have played some roles in maintaining the $\beta$ gyres, as has been discussed in Fiorino and Elsberry (1989). Over all, the results in this section demonstrate the difficulty and uncertainty in obtaining a reasonable $\beta$-gyre structure.

\section{Summary}

In this paper, we have employed the potential vorticity PV diagnostics of the GFDL hurricane model output to study the hurricane's influence on the erivironment and to understand how a hurricane may influence its own evolution by creating PV anomalies in the environment, which in turn affect the hurricane. Two sets of numerical experiments are conducted: one includes an initialized hurricane vortex using the initialization package developed by Kurihara et al. (1995), the other excludes the storm. Case studies of Hurricanes Gilbert (1988), Bob (1991), and Andrew (1992) are performed, and results from Bob are presented in detail.

The difference of PV between the hurricane and nonhurricane experiments are analyzed to understand the storm's influence on its environment. The result indicates that the areal extent of the hurricane's influence on its environmental PV field is far more extensive in the upper troposphere than in the lower troposphere. The PV budget analysis of Bob shows that the condensational heating within the vortex redistributes the PV, causing a PV sink in the upper part of the vortex and a PV source in the lower part. This tendency is com- 

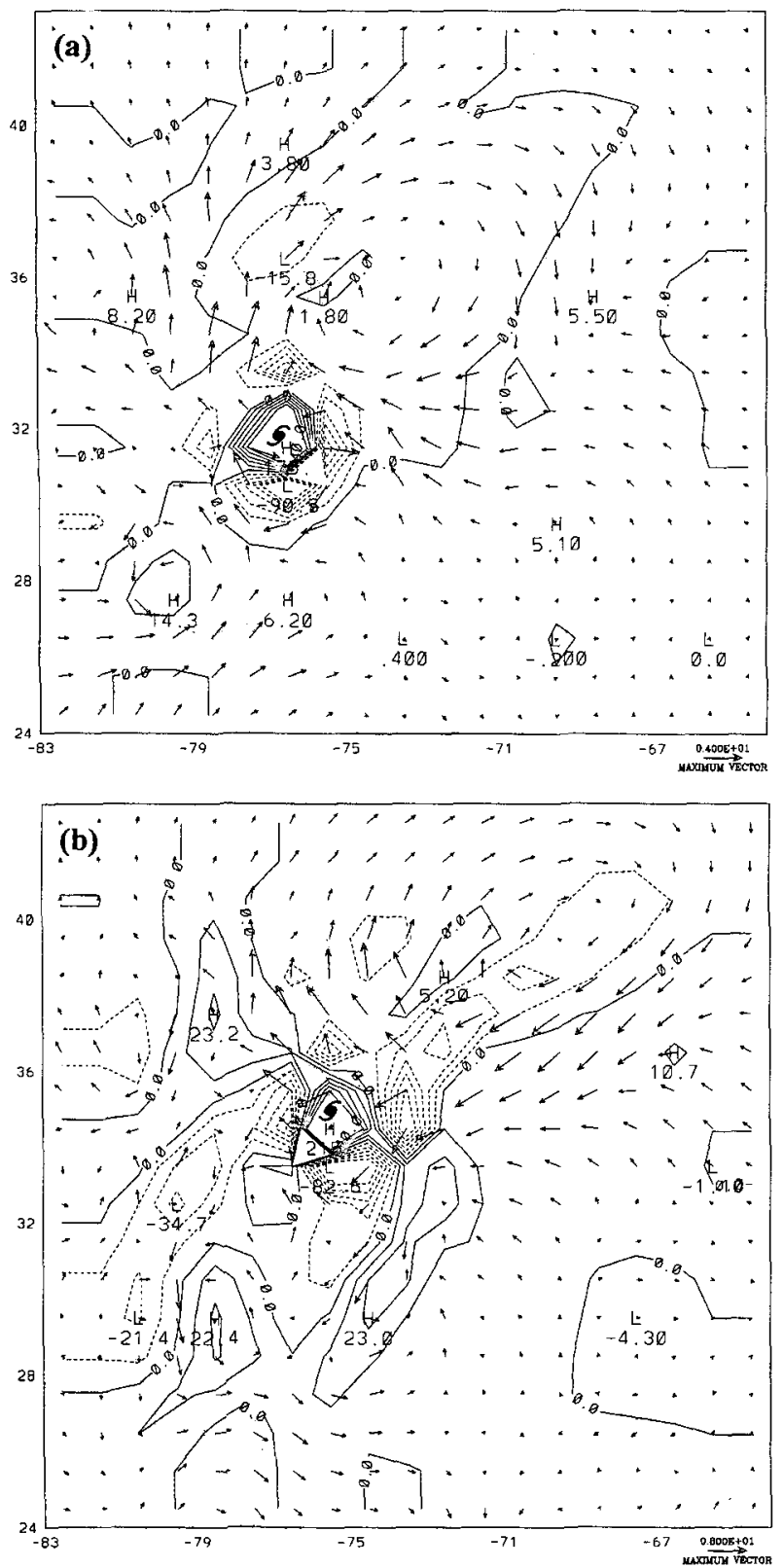

FIG. 12. The asymmetric component (relative to the storm center) of the potential vorticity perturbation and wind fields at (a) 1200 UTC 18 August (12-h forecast) and (b) 0000 UTC 19 August (24-h forecast). Values are represented in units of $0.01 \mathrm{PVU}$ with a contour interval of 0.1 PVU. The positive PV anomaly larger than 0.5 PVU at the storm center is not plotted to avoid bull's-eyes. All positive (negative) values are represented by solid (dashed) lines. The maximum wind vector plotted is 4 (or 8 ) $\mathrm{m} \mathrm{s}^{-1}$ for (a) [or (b)]. The hurricane center is indicated by the hurricane symbol.

pensated for largely, but not entirely, by the upward transport of high-PV air from the lower levels to the upper levels. These two terms are major terms in the PV budget in the vortex region, and the net effect contributes to the increase of the negative upper-level PV anomaly during the vortex intensification period. This result indicates that the diabatic heating effect plays a crucial role in the evolution of the PV field in hurricanes. It also suggests the importance of accurate representation of the heating profile in hurricane models.

It is also shown that the negative upper-level PV anomaly above Bob is exported by the outflow from the vortex region to the surroundings and later by the large-scale flow as well. Through the use of piecewise PV inversion under the nonlinear balanced condition, we show that in the middle stage of the 72 -h forecast about $1-3 \mathrm{~m} \mathrm{~s}^{-1}$ of the $850-300 \mathrm{mb}$ layer mean steering wind is attributable to the upper negative PV anomaly in the $300-100 \mathrm{mb}$ layer. The results from Bob's case study support the theory proposed in WEM93 that the hurricane motion can be affected by the flow associated with the negative upper-level PV anomaly diabatically generated above the hurricane. This result also demonstrates the need of enhanced observations and accurate analysis and prediction in the upper troposphere for improving hurricane track forecasts. Indeed, our experience from actual hurricane prediction experiments suggests that the model's capability for hurricane intensity forecasts, especially at the early stages of the storm development, is also sensitive to the quality of the initial analyses in the upper troposphere. Hence, more thought needs to be given to how to efficiently enhance observations in the upper troposphere and on how to make use of the better quality data in improving the hurricane model forecast.

Two other cases studied (Andrew and Gilbert) also showed the generation of a negative upper-tropospheric PV anomaly above the storms due to the effect of condensational heating. But their results do not show significant influences of the diabatically generated negative upper-level PV anomaly on storm movement. Whether this effect is distinct or not appears to be case dependent. More cases need to be studied to show if the influence from the upper PV anomaly on storm motion tends to be stronger for storms embedded in a more baroclinic environment. How to quantify this effect and to apply this thinking to improve actual hurricane forecasts remains a challenge for future research.

On the other hand, three different approaches have been taken to investigate the $\beta$ effect. When the asymmetric flow is obtained by excluding the axisymmetric flow relative to the storm center, well-defined $\beta$ gyres are found in the first 24-h integration of Bob. However, no conclusive evidence of the $\beta$ effect is found for other storms or by other methods. Whether we are able to detect the $\beta$ effect or not appears to be case dependent and related to how the axisymmetric component of the hurricane vortex is removed. This study indicates the difficulty in obtaining the $\beta$ gyres even when the highresolution GFDL hurricane model output is used. We feel that it is necessary to examine this effect using a high-resolution modeling and high quality observa- 
tional studies, with a view toward establishing the actual distribution of PV gradient in the atmosphere.

Finally, it should be kept in mind that even though the signals investigated in this work are on the order of a few meters per second, yet practically a few meters per second of error in estimating the hurricane motion can result in a few hundred $\mathrm{km}$ of forecast storm track error in one day. Therefore, both of the effects discussed in this paper can be crucial for hurricane track forecasts. There is a need for continuing research on the above-mentioned problems.

Acknowledgments. The authors would like to thank Morris Bender and Robert Tuleya for their helpful suggestions and kind technical assistance in running the GFDL hurricane model during this work. The authors also thank Jerry Mahlman, Morris Bender, Jeffrey Anderson, Steve Garner, and John Molinari for their helpful remarks. Credit is also given to Jeffrey Varanyak for drafting assistance. A portion of this work was carried out while the first author was affiliated with the Program in Atmospheric and Oceanic Sciences at Princeton University. The first author is partially supported through the National Science Council of Taiwan by Grants NSC84-2111-M-002-042 and NSC85-2111M-002-014-AP1.

\section{REFERENCES}

Bender, M. A., R. E. Tuleya, and Y. Kurihara, 1987: A numerical study of the effect of island terrain on tropical cyclones. Mon. Wea. Rev., 115, 130-155.

- R. J. Ross, R. E. Tuleya, and Y. Kurihara, 1993: Improvements in tropical cyclone track and intensity forecasts using GFDL initialization system. Mon. Wea. Rev., 121, 2046-2061.

Chan, J. C.-L., and W. M. Gray, 1982: Tropical cyclone movement and surrounding flow relationship. Mon. Wea. Rev., 110, 13541376.

the beta-effect in tropical cyclone motion. Part I: Zero mean flow. J. Atmos. Sci., 44, 1257-1264.

Davis, C. A., and K. A. Emanuel, 1991: Potential vorticity diagnostics of cyclogenesis. Mon. Wea. Rev., 119, 1929-1953.

Elsberry, R. L., B. C. Diehl, J. C.-L. Chan, P. A. Harr, G. J. Holland, M. Lander, T. Neta, and D. Thom, 1990: ONR tropical cyclone motion research initiative: Field experiment summary. Tech. Rep. NPS-MR-91-001, Naval Postgraduate School, Monterey, $\mathrm{CA}, 107 \mathrm{pp}$.

Fiorino, M., and R. L. Elsberry, 1989: Some aspects of vortex structure related to tropical cyclone motion. J. Atmos. Sci., 46, 975-990.

Haynes, P. H., and M. E. McIntyre, 1987: On the evolution of vorticity and potential vorticity in the presence of diabatic heating and frictional or other forces. J. Atmos. Sci., 44, 828-841.

Holland, G. J., L. M. Leslie, and B. C. Diehl, 1992: Comments on "The detection of flow asymmetries in the tropical cyclone environment."' Mon. Wea. Rev., 120, 2394-2400.

Hoskins, B. J., M. E. Mclntyre, and A. W. Robertson, 1985: On the use and significance of isentropic potential-vorticity maps. Quart. J. Roy. Meteor. Soc., 111, 877-946.
Kurihara, Y., 1973: A scheme of moist convective adjustment. Mon. Wea. Rev., 101, 547-553.

_- and M. A. Bender, 1980: Use of a movable nested mesh. model for tracking a small vortex. Mon. Wea. Rev., 108, 1792-- 1809.

- C. L. Kerr, and M. A. Bender, 1989: An improved numerical scheme to treat the open lateral boundary of a regional model. Mon. Wea. Rev., 117, 2714-2722.

—- M. A: Bender, R. E. Tuleya, and R. Ross, 1990: Prediction experiments of Hurricane Gloria (1985) using a multiply nested movable mesh model. Mon. Wea. Rev., 118, 2185-2198.

- - - and R. J. Ross, 1993: An initialization scheme of hurricane models by vortex specification. Mon. Wea. Rev., 121, 2030-2045.

,,-- R. E. Tuleya, and R. J. Ross, 1995: Improvemer.t in the GFDL hurricane prediction system. Mon. Wea. Rev., 123, 2791 2801.

Lacis, A. A., and J. E. Hansen, 1974: A parameterization for the absorption of solar radiation in the earth's atmosphere. J. Atmos. Sci., 31, 118-133.

Mellor, G. L., and T. Yamada, 1974: A hierarchy of turbulence closure models for planetary boundary layers. J. Atmos. Sci., 31, $1791-1806$.

Molinari, J., S. Skubis, and D. Vollaro, 1995: External influences on hurricane intensity. Part III: Potential vorticity structure. J. Atmos. Sci., 52, 3593-3606.

Reeder, M. J., R. K. Smith, and S. J. Lord, 1991: The detection of flow asymmetries in the tropical environment. Mon. Wea. Rev., $119,848-855$.

$-\frac{1}{2400}$, and,- 1992 : Reply. Mon. Wea. Rev., 120, 2398-

Ross, R. J., and Y. Kurihara, 1992: A simplified scheme to :simulate asymmetries due to the beta effect in barotropic vortices. $J$. Atmos. Sci., 49, 1620-1628.

- , and - 1995: A numerical study of influences of Hurricane Gloria (1985) on the environment. Mon. Wea. Rev., $123,332-346$.

Schwarzkopf, M. D., and S. B. Fels, 1991: The simplified exchange method revisited: An accurate, rapid method for computation of infrared cooling rates and fluxes. J. Geophys. Res., 96, 90759096.

Shapiro, L. J., 1992: Hurricane vortex motion and evolution in a three-layer model. J. Atmos. Sci., 49, 140-153.

Tuleya, R. E., 1994: Tropical storm development and decay: Sensitivity to surface boundary conditions. Mon. Wea. Rev., 122, 291-304.

_- M. A. Bender, and Y. Kurihara, 1984: A simulation study of the landfall of tropical cyclones using a movable nested-mesh model. Mon. Wea. Rev., 112, 124-136.

Ulrich, W., and R. K. Smith, 1991: A numerical study of tropical cyclone motion using a barotropic model. II: Motion in spatially-varying large-scale flows. Quart. J. Roy. Meteor. Soc., 117, $107-124$.

Wu, C.-C., and K. A. Emanuel, 1993: Interaction of a baroclinic vortex with background shear: Application to hurricane movement. J. Atmos. Sci., 50, 62-76.

_- and -1994 : On hurricane outflow structure. J. Atrios. Sci., 51, 1995-2003.

- $\ldots$, and — 1995a: Potential vorticity diagnostics of hurricane movement. Part I: A case study of Hurricane Bob (1991). Mon. Wea. Rev., 123, 69-92.

$\longrightarrow$, and -1995 b: Potential vorticity diagnostics of hurricane movement. Part II: Tropical Storm Ana (1991) and Flurricane Andrew (1992). Mon. Wea. Rev., 123, 93-109. 